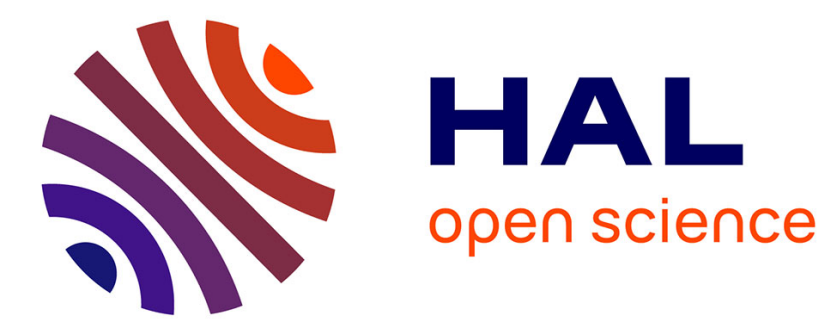

\title{
Active optics with a minimum number of actuators
}

Gerard Lemaitre

\section{To cite this version:}

Gerard Lemaitre. Active optics with a minimum number of actuators. Advanced Optical Technologies, 2014, 3 (3), pp.223 - 249. 10.1515/aot-2014-0014 . hal-02058793

\section{HAL Id: hal-02058793 https://hal.science/hal-02058793}

Submitted on 6 Mar 2019

HAL is a multi-disciplinary open access archive for the deposit and dissemination of scientific research documents, whether they are published or not. The documents may come from teaching and research institutions in France or abroad, or from public or private research centers.
L'archive ouverte pluridisciplinaire HAL, est destinée au dépôt et à la diffusion de documents scientifiques de niveau recherche, publiés ou non, émanant des établissements d'enseignement et de recherche français ou étrangers, des laboratoires publics ou privés. 


\title{
Review Article
}

\author{
Gerard R. Lemaitre*
}

\section{Active optics with a minimum number of actuators}

\begin{abstract}
Optics for astronomy implies powerful developments of active and adaptive optics methods applied to instrumentation from X-rays to the near infrared for the design of telescopes, spectrographs, and coronagraph planet finders. This presentation particularly emphasizes the development of active optics methods. Highly accurate and remarkably smooth surfaces from active optics methods allow new optical systems that use highly aspheric and non-axisymmetric - freeform - surfaces. Depending on the goal and performance required for a deformable optical surface, elasticity theory analysis is carried out either with small deformation thin plate theory, large deformation thin plate theory, shallow spherical shell theory, or the weakly conical shell theory. A mirror thickness distribution is then determined as a function of associated bending actuators and boundary conditions. For a given optical shape to generate, one searches for optical solutions with a minimum number of actuators.
\end{abstract}

Keywords: active optics; astronomical optics; elasticity theory; optical design; X-ray optics.

OCIS codes: 010.1080; 220.0220; 050.1950; 220.1000; 220.1250; 340.7470; 350.1260.

DOI 10.1515/aot-2014-0014

Received February 27, 2014; accepted April 17, 2014

\section{Introduction}

Active optics methods are reviewed using various concepts of deformable astronomical optics, which have been elaborated in our optical laboratory of the Marseille Observatory - LOOM - since the 1970s, and also at other

*Corresponding author: Gerard R. Lemaitre, Laboratoire d'Astrophysique de Marseille, LOOM, Aix Marseille Université and CNRS, 38 rue Fréderic Joliot-Curie, F-13388 Marseille CX 13, France, EU, e-mail: gerard.lemaitre@lam.fr

www.degruyter.com/aot

(C) 2014 THOSS Media and De Gruyter astronomical institutes around the world. Elasticity analyses and optical designs allow the optimization of substrate geometry with appropriate boundary conditions for applying bending forces. For materials having a linear stress-strain relationship, such as glass and some metal alloys, these methods provide accurate optical deformation modes, which fully satisfy diffraction-limited criteria.

\section{Elasticity methods and active optics}

Among various field applications of active optics methods, are the following:

- stress figuring processes of optical surfaces with large aspherization capability,

- in situ shaping processes of telescope mirrors with large aspherization capability,

- in situ reshaping and alignment of large telescope optics with closed-loop wavefront sensors,

- variable curvature - or zoom - mirrors for field cophasing of telescope arrays and two-arm interferometers,

- aberration-corrected diffraction gratings made by replication from actively aspherized submasters,

- deformable compensators for photosynthesis holographic recording of corrected gratings.

Optimal elasticity designs led us to consider various substrate classes: constant thickness, quasi-constant thickness, variable thickness, and hybrid thickness. The elasticity theory of thin plates provides accurate results when the curvature of the middle surface of the substrate is small, whereas the theory of shallow shells is required for more curved substrates, say for mirrors faster than $\mathrm{f} / 3$. The large deformation theory allows accurate designs for variable curvature mirrors with large zoom ranges. Results from the new theory of weakly conical shells are presented for X-ray telescope using tubular mirrors.

A book on Active Optics Methods including analytic developments of elasticity theory and results from optical testing has been recently published by the author [1]. 


\section{Origins of Active Optics - the Schmidt telescope}

To avoid surface discontinuities or ripple errors caused by local retouching with small figuring laps, B. Schmidt, inventor of the wide-field telescope (Figures 1 and 2), suggested around 1932 that an aspheric surface could be obtained by elastic relaxation after spherical figuring with a full aperture lap. Schmidt's idea of obtaining smooth aspheric surfaces - i.e., free from high spatial frequency errors - remained of great potential interest and was also advocated by H. Chrétien in his book Combinaisons Optiques.

\subsection{Single-zone loading method}

Although B. Schmidt partly experimented its single-vacuum-zone method (Figure 3) when making his f/1.75 telescope in 1931, he finished the 36-cm-corrector plate with the local-retouch method for which he was a renowned expert who aspherized many telescope mirrors for European observatories. The origin of aspherizing a corrector plate via complete active optics goes back to E. Everhart

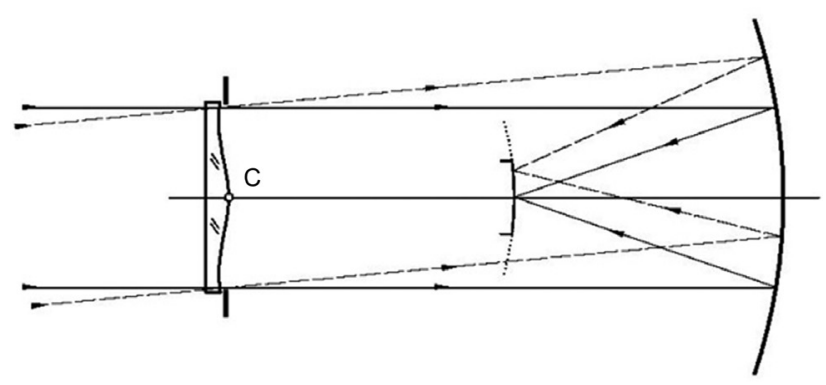

Figure 1 Schmidt wide-field telescope design.

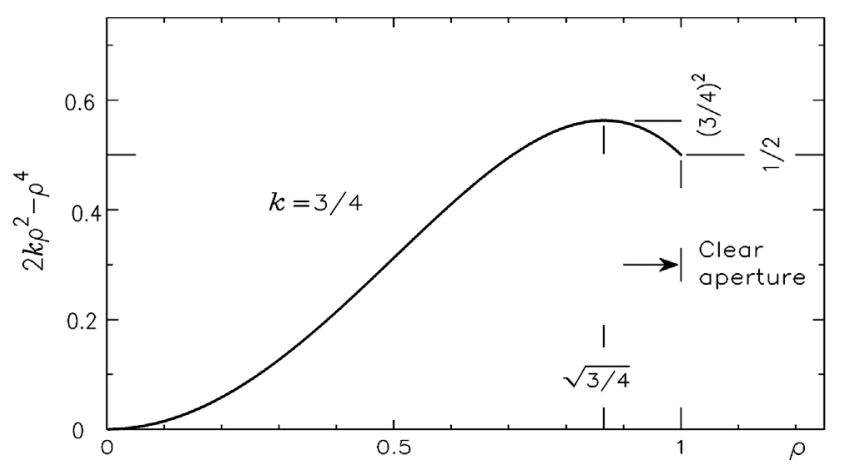

Figure 2 Kerber profile of Schmidt corrector plate. Balance of slopes minimizes spherochromatism.
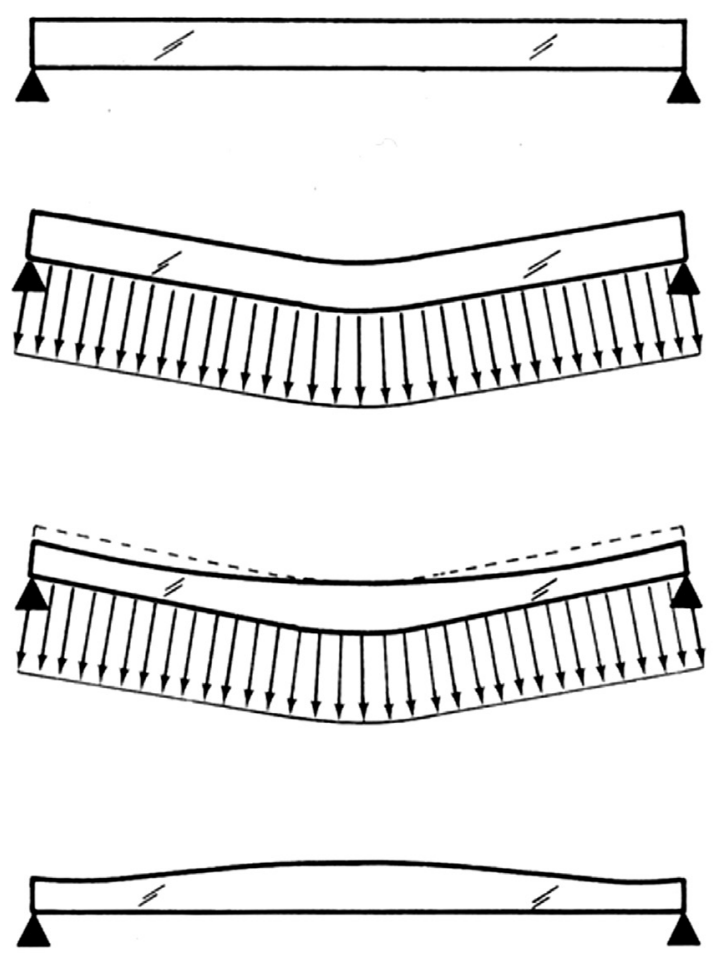

Figure 3 Corrector plate aspherization by single-zone loading method with spherical full-size lap.

[2] in 1965 who applied the method in making a 29-cm corrector plate for a Wright-type telescope at $\mathrm{f} / 5$. He solved for and published the design elasticity parameters derived from the fourth-derivative Poisson equation of thin plate theory for constant thickness. The flexure $z(r)$ is a biquadratic polynomial solution of

$$
\Delta^{2} \Delta^{2} z(r)+q / D=0,
$$

where $\Delta^{2}$ is the Laplacian operator, $r$ is the current radius, $q$ the uniform load applied over the cylindrical plate when supported at its edge, and $D$ the rigidity a constant proportional to the third power of the plate thickness $t[1]$.

\subsection{Double-zone loading method}

Kerber's law (1886) [1] for achromatic doublet lenses also applies to an aspheric singlet plate. It states that residual sphero-chromatism is minimal when the aspheric profile of a refractive corrector plate provides an algebraic balance of the slopes [1], i.e., an aspheric surface of the form

$$
z=z_{0}\left(3 \rho^{2} / 2-\rho^{4}\right)
$$



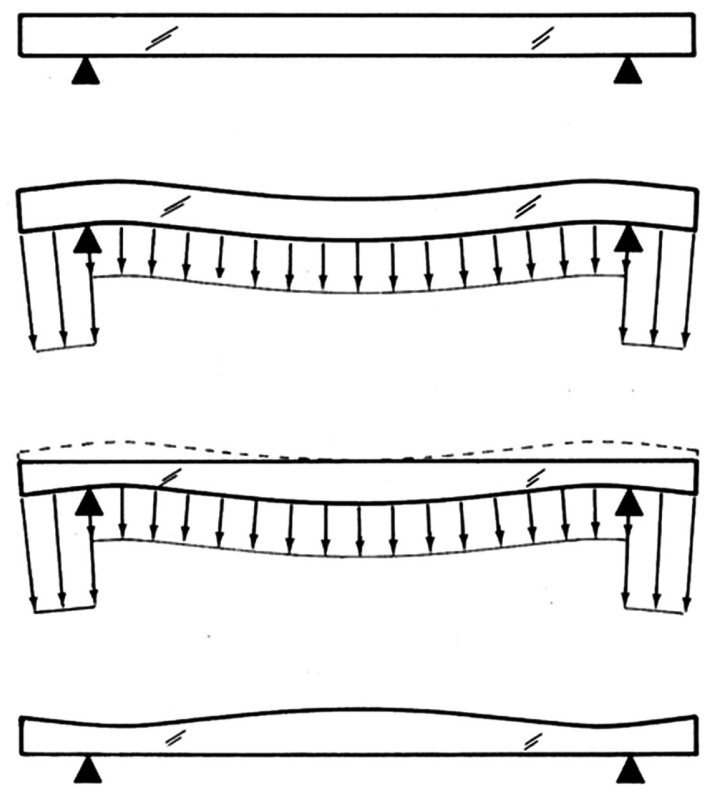

Figure 4 Corrector plate aspherization by double-zone loading method with flat full-size lap.

where $z_{0}$ is a constant and $\rho \in[0,1]$ the dimensionless aperture radius $r / r_{\max }$. Hence, from Eq. (2), the null-power zone - no deviation of a ray - is located at the optical plate height $=\sqrt{ } 3 / 2=0.866$.

The above single-zone loading method does not provide a flexure expressed in Eq. (2). When the flexural $\rho^{4}$ term is conveniently set to compensate for third-order aberration, it generates a $\rho^{2}$-term much larger than that in
Eq. (2). This requires use of a spherical lap of convenient curvature to obtain a Kerber profile. This method presents technical difficulties caused by the lack of control of the lap radius of curvature during polishing and thus control of the null-power zone location.

Because of these difficulties, Lemaitre proposed the double-zone loading method [3] with full-size plane figuring laps (Figure 4). Solving Eq. (1) with two concentric uniform loads allows this method to directly provide a flexure of opposite sign to that of Eq. (2). Developments at LOOM have led to about a hundred corrector plates from $f / 3: 5$ up to $f / 1: 1$ for telescopes, spectrographs, and embarked cameras up to $62-\mathrm{cm}$ clear aperture [1] (Figures 5 and 6). A Schott BK7 62-cm clear aperture corrector plate for the Franco-Belgium Schmidt telescope (Figure 7) at the Haute Provence Observatory (OHP) was aspherized with this method to replace the original plate.

\section{Variable curvature mirrors (VCMs) for telescope arrays and Fourier interferometers $Z=A_{20} r^{2}$}

Telescope array interferometers and infrared Fourier transform interferometers require use of variable curvature mirrors (VCM). These mirrors are located at the focal surface of retro-reflective devices called cat's eye systems (Figure 8). Such optical systems are mounted on carriage translators to provide the optical path compensation due
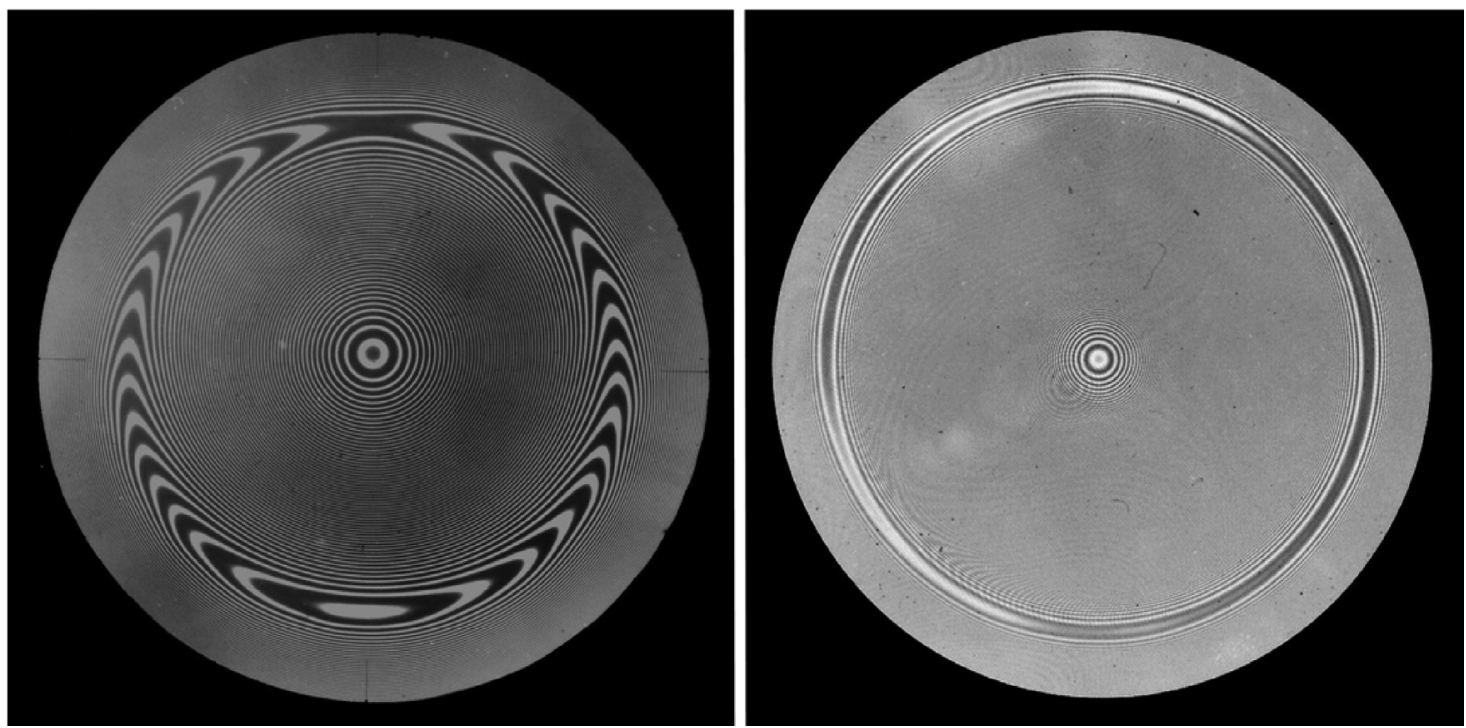

Figure 5 He-Ne interferograms of equal-thickness fringes of plates aspherized by the double-zone method. Left: 10-cm clear aperture plate at $\mathrm{f} / 2$. Right: $10-\mathrm{cm}$ clear aperture plate at $\mathrm{f} / 1.2$. 

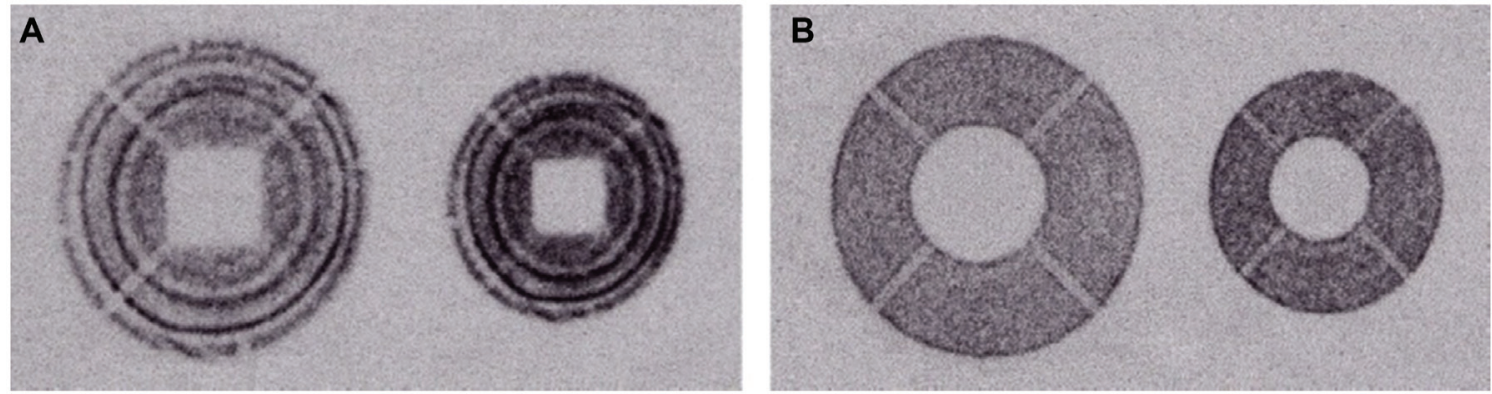

Figure 6 Intra-focus patterns of a star obtained with $62-\mathrm{cm}$ aperture plates, aspherized (A) by zonal retouch and (B) by stress figuring, of the 62/90-cm Franco-Belgium Schmidt telescope (LOOM).

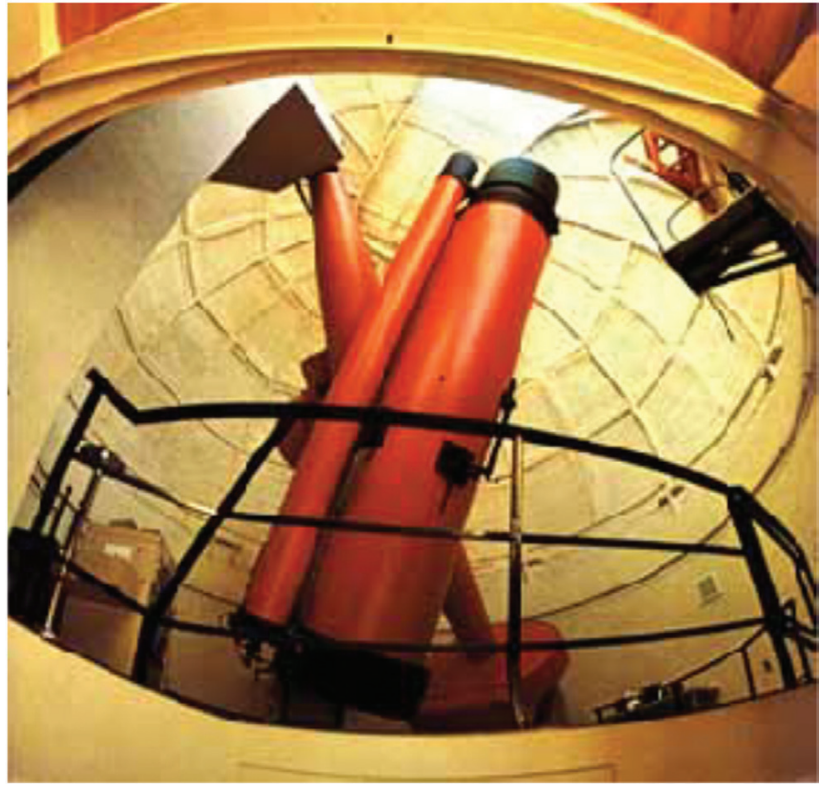

Figure 7 Franco-Belgium 62/90-cm Schmidt telescope at f/3.3 (Haute Provence Observatory and LOOM). to the Earth rotation during observations. While translation of the cat's eye system maintains equality of the on-axis optical path - on-axis cophasing - the off-axis optical path compensation - field-of-view compensation or field cophasing - is achieved by a VCM. This mirror maintains the output pupil conjugation of the delay line at a fixed location before the recombination laboratory of the telescope array.

These active mirrors generate a first-order pure curvature mode - here called $C v 1$ mode - of the simple form $z=A_{20} r^{2}$, where $A_{20}$ is a constant of length dimension.

Elasticity theory can provide highly deformable mirrors satisfying diffraction-limited quarterwave criterion by use of metal alloys having linear stress-strain relationships and a single actuator as presented hereafter.

Load configurations with constant thickness distributions - CTD class - of the substrate are generated with accuracy from a bending moment uniformly distributed along the circular mirror edge (Figure 9). Because of the practical complexity to generate the bending moments distributed at the mirror edge, these configurations have not yet been developed.

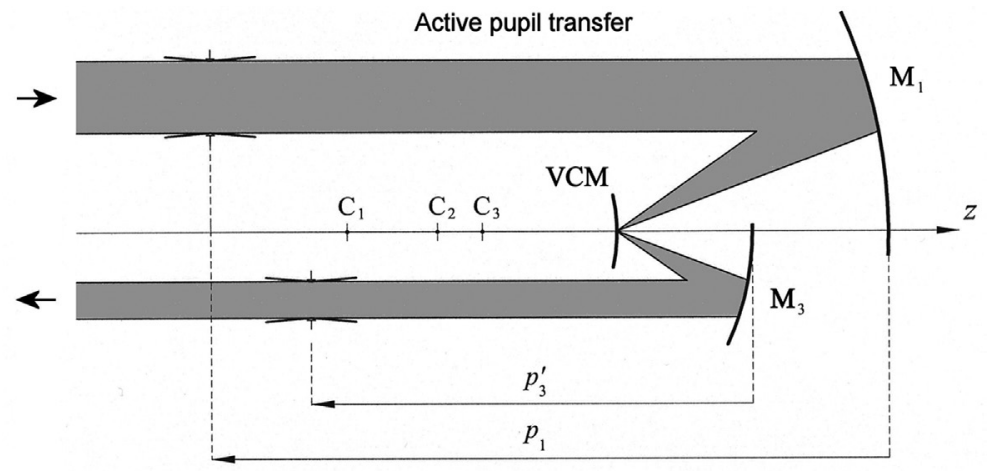

Figure 8 Optical design of a retro-reflective cat's eye system. The VCM is mounted at the focal surface of $M_{1}$ mirror and provides the optical path compensation of the field-of-view or field cophasing. This allows control of the location $p_{3}^{\prime}$ of the output pupil with respect to the input pupil at $p_{1}$. This active pupil transfer is aplanatic if $M_{1}$ and $M_{3}$ are Mersenne confocal paraboloids. 


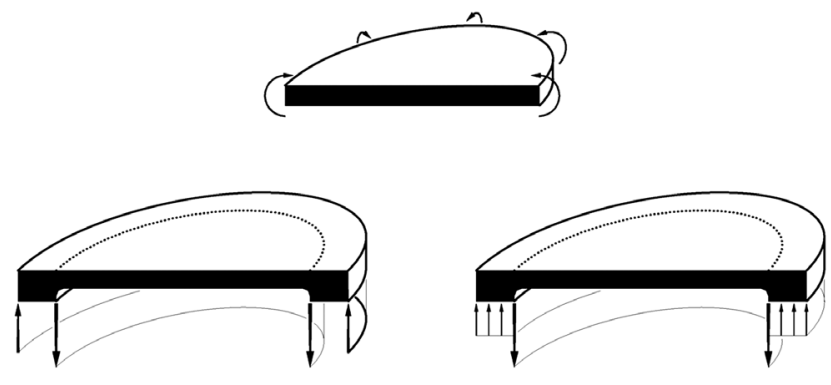

Figure 9 VCM configurations with constant-thickness distributions - CTD class.

Three more practicable loading configurations designed with variable thickness distributions - VTD class - have been found by Lemaitre $[1,4]$ and developed at LOOM. The first configuration, a cycloid-like form VCM, is associated with a uniform load. The other two, called tulip form VCMs, are associated with a central force (Figure 10).

Thin plate elasticity theory provides CTD or VTD configurations that generate $C v 1$ modes of diffraction-limited quality for zoom ranges extending, say, from $f / \infty$ to $f / 5$. For obtaining larger zoom ranges, the elasticity theory of large deformations has been applied by Ferrari $[6,7]$ to the design of cycloid-like-form and tulip-form VCMs. The two forms shown at the top of Figure 10, with load reaction at the edge, were built in a stainless steel metal substrate by selecting the quenched alloy FeCr13, which exhibits a highly linear stress-strain relationship. The mirror design parameters are $16 \mathrm{~mm}$ aperture, $300 \mu \mathrm{m}$ central thickness, and $400 \mu \mathrm{m}$ maximum flexural sag typically at 7 bars loading. The reflective zone is simply supported by

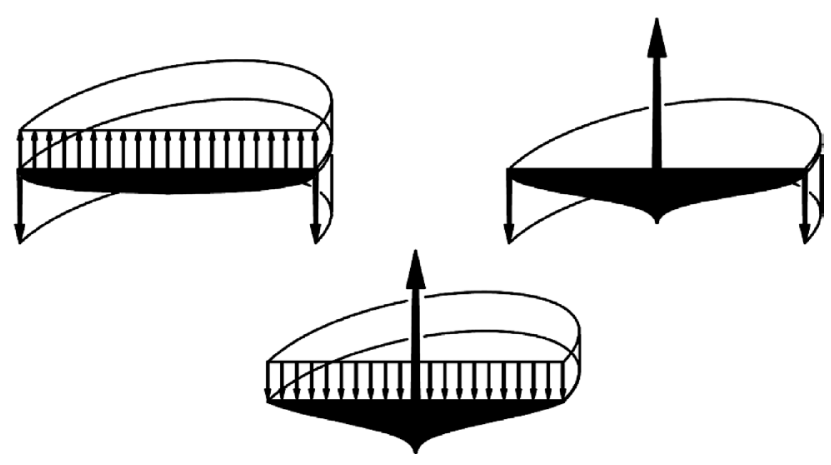

Figure 10 VCM configurations with variable thickness distributions - VTD class. Thicknesses: Up-left: $t / t_{0}=\left(1-\rho^{2}\right)^{1 / 3}$ uniform loading and reaction at edge. Up-right: $t / t_{0}=\left(-\ln \rho^{2}\right)^{1 / 3}$ central force and reaction at edge. Down: $t / t_{0}=\left(\rho^{2}-\ln \rho^{2}-1\right)^{1 / 3}$ uniform loading and central force $[4,5]$. Because the two latter configurations show an infinite thickness at the center, one can define the central thickness $t_{0}$ as that corresponding to a very small radius, e.g., $\rho=10^{-3}$, in agreement with Rayleigh's quarter wave criterion. a $25-\mu \mathrm{m}$ thin outer cylinder linked to a rigid ring. These three parts - reflective zone, outer cylinder, and rigid ring - are made in a single or holosteric piece. Interferometric tests were carried out with respect to spherical calibers of incremented curvatures (Figures 11-13).

The on-axis path cophasing of the VLTI array uses translating delay lines by Derie et al. [8] that compensate for the path variation caused by diurnal rotation. Each delay line is equipped with a VCM at the focal plane of a Ritchey-Chretien cat's eye system for the off-axis path cophasing or field cophasing (Figures 14 and 15). The implementation of VCMs on ESO VLTI is eight VCMs on eight delay lines (four UTs, $8 \mathrm{~m}+$ four ATs, $2 \mathrm{~m}$ ) and eight VCMs on four ATs for the phase reference imaging combiner PRIMA.

\section{Mirrors generating single aberration modes with a minimum number of actuators}

We investigated four thickness class distributions for mirror substrates and load distributions able to separately generate the primary aberration modes Sphe3, Coma3, and Astm3 (Lemaitre [1, 9]) and using a maximum of two or three actuators. These thickness classes are the following: constant thickness distribution (CTD), quasi-constant thickness distribution (q-CTD), variable thickness distribution (VTD), and a combination of them: the hybrid thickness distribution (HTD).

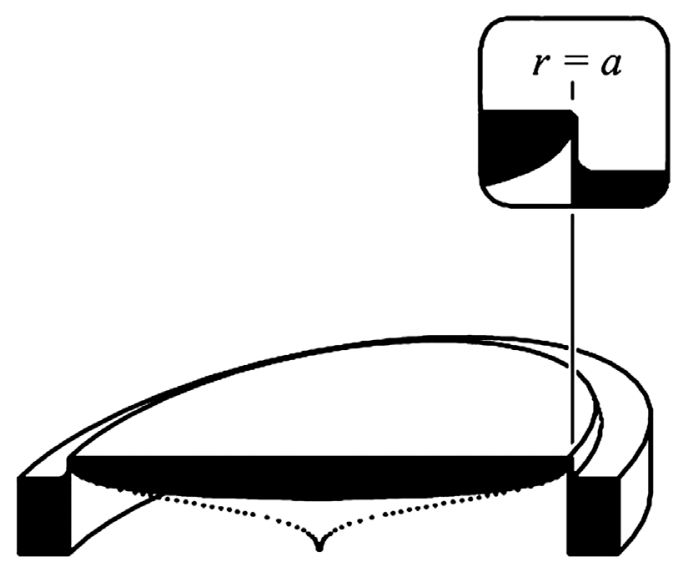

Figure 11 Holosteric design of cycloid-like form (full line) and tulip form (dotted line) VCMs. The outer thin collar link ensures equivalent boundaries to a simply-supported edge. 


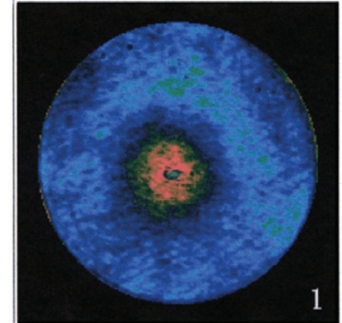

$f / \infty$

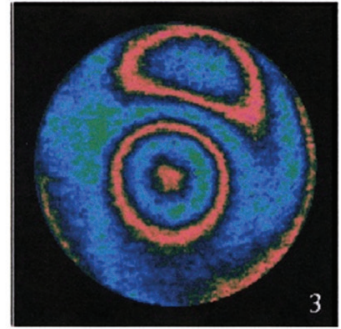

f/ 8.1

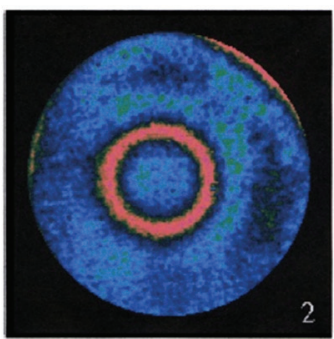

f / 13

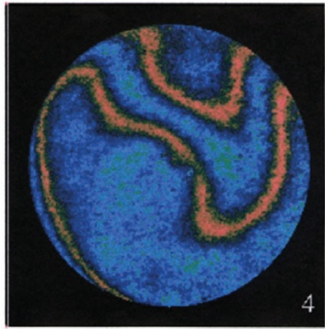

$f / 4.4$

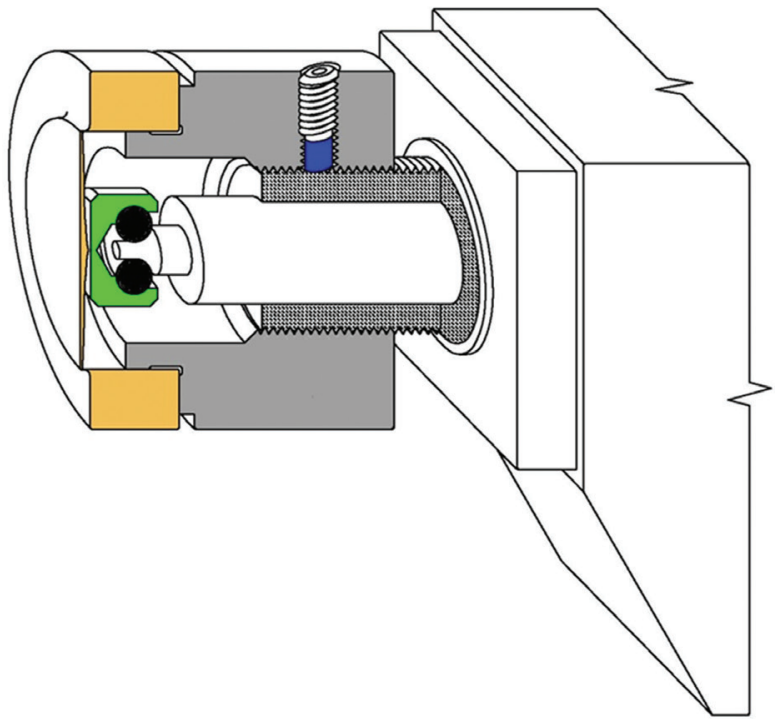

Figure 12 He-Ne interferometric null tests with respect to spherical calibers of a tulip form VCM actuated at its center by control motorized push drive and ball pad. Aperture diameter $16 \mathrm{~mm}$, central thickness $t_{0}=320 \mu \mathrm{m}$, maximum flexural sag $227 \mu \mathrm{m}$, mirror zoom range $\mathrm{f} / \infty$ to $\mathrm{f} / 4.4$. The peak-to-valley wavefront error is lower than $600 \mathrm{~nm}$ for all the zoom range (LOOM).

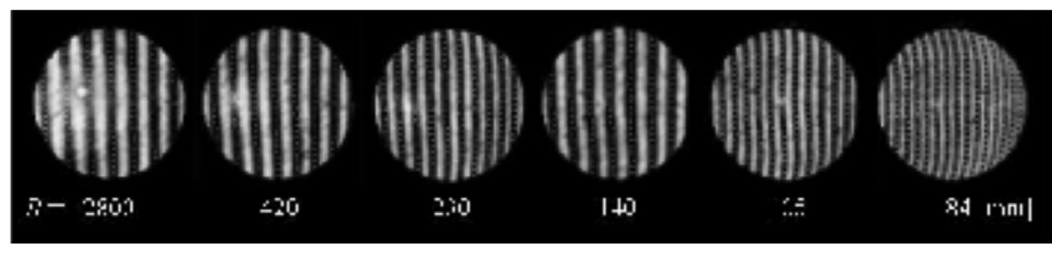

Figure 13 He-Ne interferometric null tests with respect to spherical calibers of one of 20 cycloid-like form VCMs made for the ESO VLTI Array. Aperture diameter $16 \mathrm{~mm}$, central thickness $t_{0}=300 \mu \mathrm{m}$, maximum flexural sag $381 \mu \mathrm{m}$, maximum load $7 \mathrm{bars}$, mirror zoom range $\mathrm{f} / \infty$ to $\mathrm{f} / 2.6$ (LOOM).

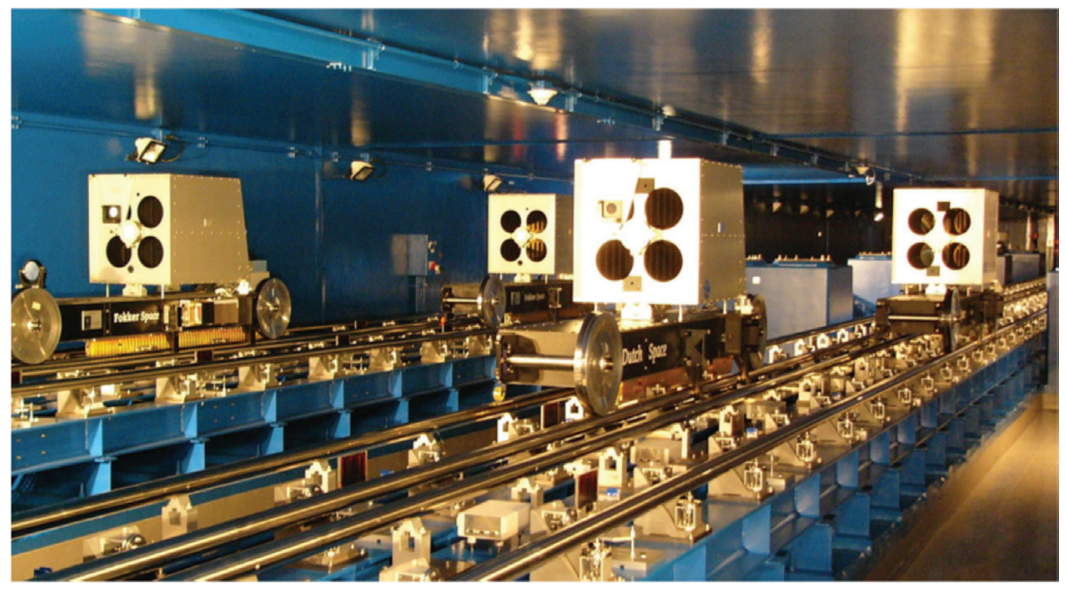

Figure 14 Four of the eight delay lines and cat's eye systems with VCMs at the Very Large Telescope Interferometer tunnel (ESO).

\subsection{Primary spherical aberration mode $z=A_{40} r^{4}$}

From these classes, several configurations were found for mirrors generating a spherical aberration mode Sphe3, i.e., a flexure of the form $z=A_{40} r^{4}$. It can be shown [4] that a uniform load applied over the mirrors' entire clear aperture is necessary to obtain an $r^{4}$ flexure, but for constant thickness plates, a uniform bending moment must 

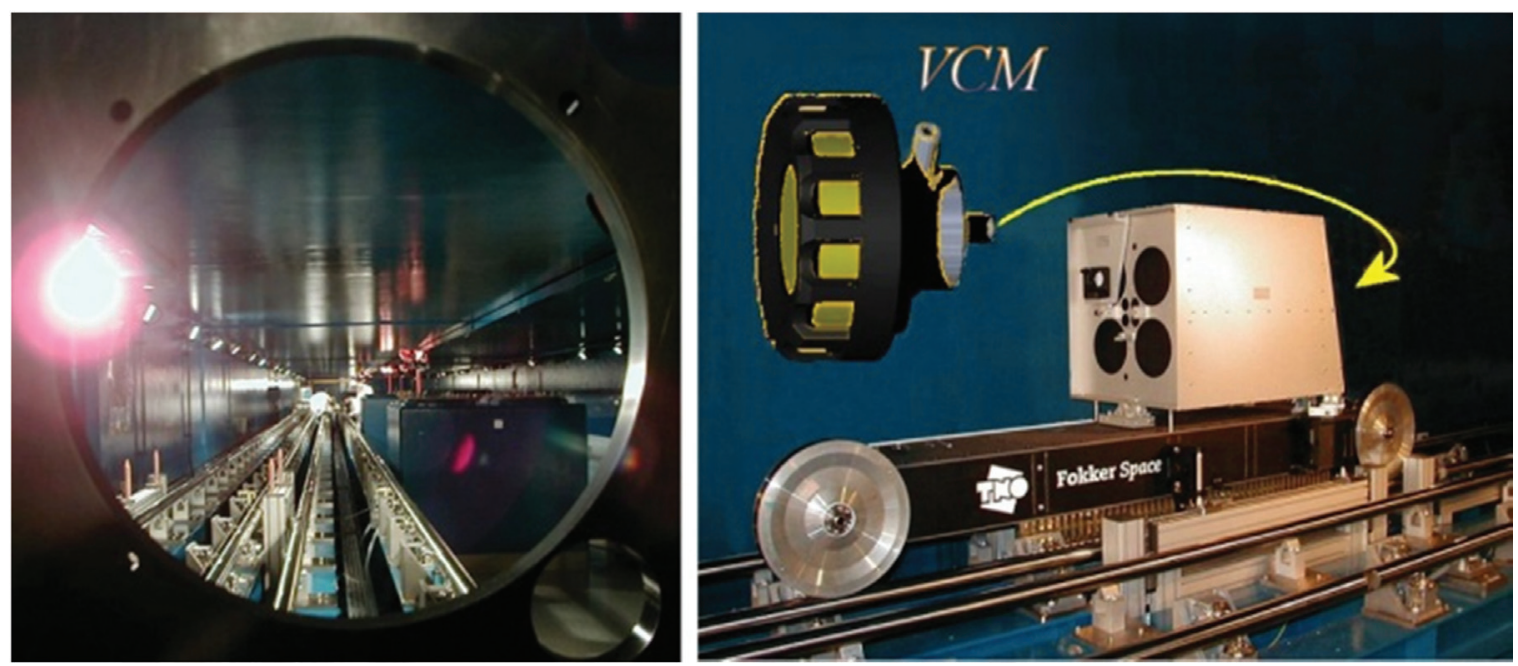

Figure 15 Left: View of the 70-m length VLTI delay lines. Right: View of the translation carriage with a cat's eye system made of an $\mathrm{f} / 2$ Ritchey-Chrétien telescope with a VCM is located at its focus (LOOM and ESO).

be applied over the outer contour to cancel the curvature term - i.e., the $r^{2}$ flexure. However, similarly to the case of the $C v 1$ mode, the CTD class provides possible theoretical configurations (Figure 16), but these lead to some difficulty for applying the bending moments. Hence, designs in the VTD class are much easier to make. Three variable thickness distributions belonging to the VTD class were found by Lemaitre in $1976[1,4]$. All of them are of tulip form - i.e., with infinite thickness at center - and associated with a central force, a uniform load, or a uniform reaction at the circular edge (Figure 17).

A tulip form $r^{4}$-deformable mirror was developed at LOOM for making the first Sphe3 aberration-corrected grating, in 1976, from a plane diffraction grating, using double-replication technique. A plane grating is first deposited on the intermediate plane-deformable tulipform submaster [1]. The final grating replica is obtained during controlled stress of the metal submaster in a FeCr13 alloy (Figure 18). Another possibility for generating a Sphe3 mode is to combine the CTD and VTD classes, thus forming a hybrid thickness distribution (HTD) [1] class (Figure 19).

\subsection{Balanced primary spherical aberration $z=A_{20} r^{2}+A_{40} r^{4}$}

A combination of $C v 1$ and Sphe 3 modes with $A_{20} A_{40}<0$ allows reduction of the stress level in the deformable substrate. These combined modes are of particular interest for
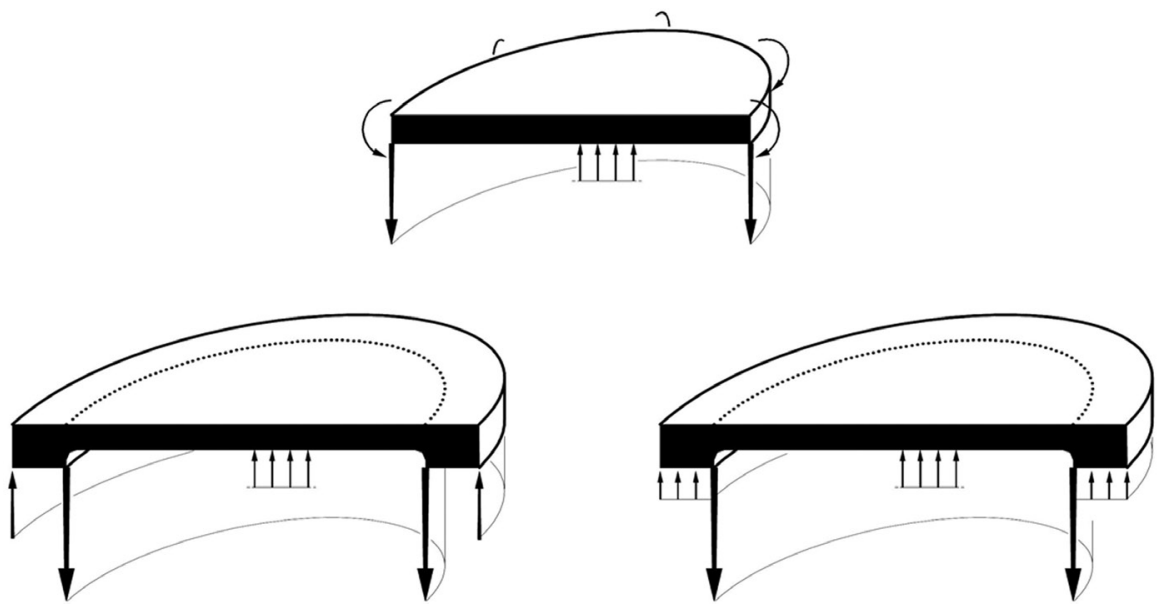

Figure 16 Configurations derived from CTD class mirrors generating Sphe3 mode. (The schematic representation of the four-force set near the center of the mirrors means a uniform load applied all over its rear surface.) 


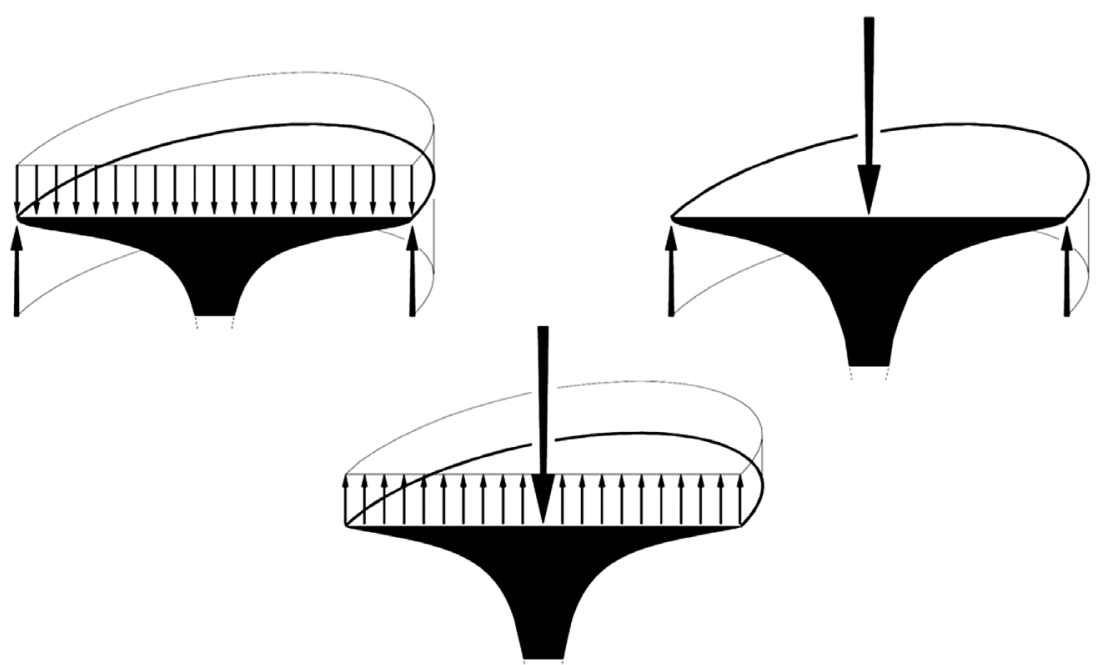

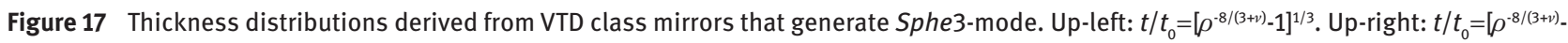
$\left.\rho^{-2}\right]^{1 / 3}$. Down: $t / t_{0}=\left[((3+v) /(1-v)) \rho^{-8 /(3+v)}-(4 /(1-v)) \rho^{-2}+1\right]^{1 / 3}$, where $v$ is Poisson's ratio.
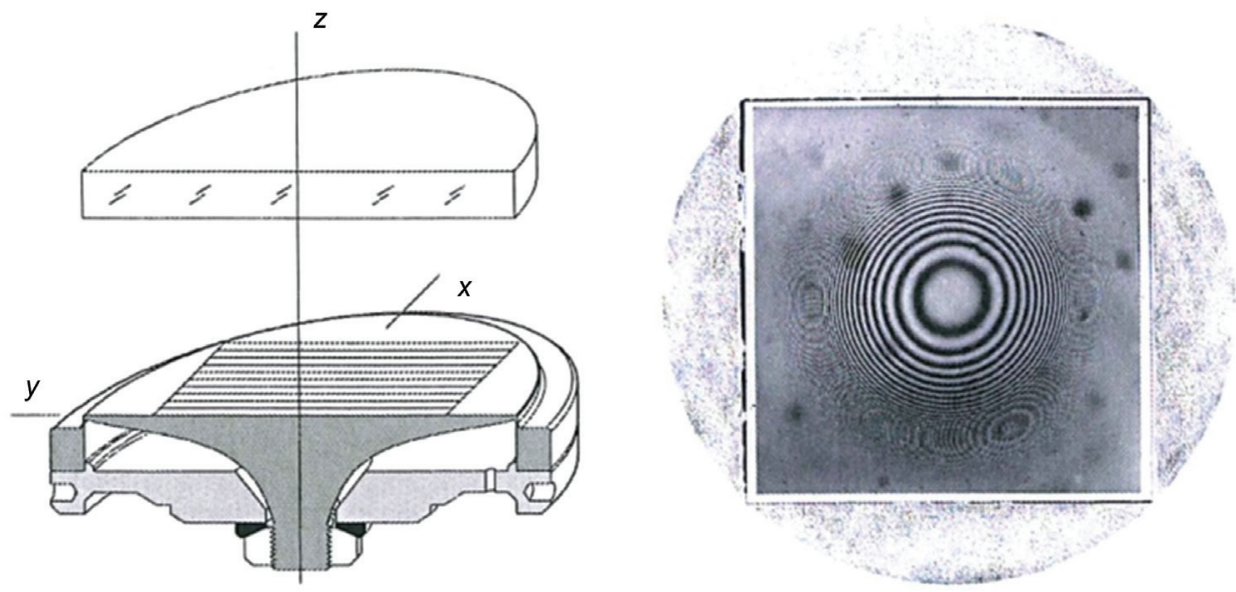

Figure 18 Making the first Sphe3 corrected grating on a Zerodur substrate using two-stage replication. Left: Active optics submaster. Right: He-Ne interferogram of final replica grating (LOOM and Jobin Yvon Horiba).
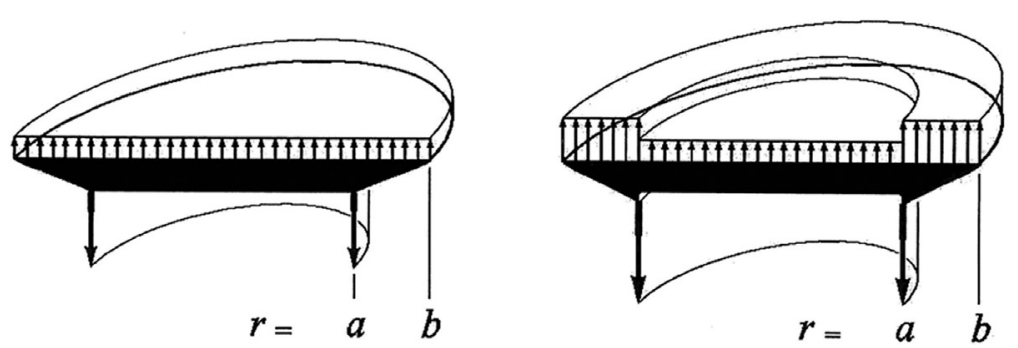

Figure 19 Two solutions for generating an $r^{4}$-flexure for a Sphe3 aberration-corrected mode derived from the HTD class mirrors.

any reflective systems such as high-throughput Schmidt cameras of spectrographs equipped with aspheric reflective gratings. Balancing $\mathrm{C} v 1$ and Sphe 3 modes also substantially improve the optical performance by minimizing residual field aberrations. Several applications and results are presented below.

\subsubsection{Reflective Schmidt systems: telescopes and spectrographs}

Without considering the tilt angle of the first mirror or grating (i.e., assuming a 100\% obstruction fictive telescope), a reflective Schmidt is an anastigmat, i.e., free from 
third-order spherical aberration, coma, and astigmatism. An important law for the design of the first element of any reflective Schmidt system was found by Lemaitre $[1,10]$ :

$\Rightarrow$ A pupil reflective surface with balanced radial variation of its local curvatures minimizes the field aberrations of any reflective Schmidt system.

This law minimizes dominating residual fifth-order astigmatism and applies to a Schmidt primary mirror such as the giant Schmidt LAMOST - as well as to imagerspectrograph camera optics using a reflective corrector or an aspherized diffraction grating.

Aspherized reflective gratings provide a very compact optical design of the camera optics (Figure 20). Deformable submasters with a built-in boundary at the edge and a quasi-constant thickness distribution (q-CTD) were developed at LOOM to generate aspherized gratings through the double replication technique (Figure 21). For central wavelengths diffracted perpendicular to the grating surface $(\beta=0)$, one can show that its best surface is axisymmetric. In this later case, from the above law on the balance of curvatures, the equation of the aspheric surface must be

$$
z=z_{0}\left(3 \rho^{2}-\rho^{4}\right)
$$

where $z_{0}$ is a constant and $\rho \in[0,1]$ the dimensionless aperture radius $r / r_{\text {max }}$. One can check from Eq. (3) that the $d^{2} z / d \rho^{2}$ values are balanced for $\rho=0$ and 1 and that the optics null-power zone - no deviation of a ray - is then located at the dimensionless radius $\rho=\mathrm{V}(3 / 2)=1.225$, i.e., outside the maximum optical aperture $2 r_{\max }$.

The q-CTD design leads to small decrease of the thickness toward the submaster edge for also correcting the next-order aberration Sphe5. Many spectrographs have benefited from these gratings (CFHT Mauna Kea, Pic du Midi, Haute Provence and Purple Mountain Observatories, Space Mission Odin-Osiris, etc). These gratings were built either for on- or off-axis mountings [1] (Figure 22).
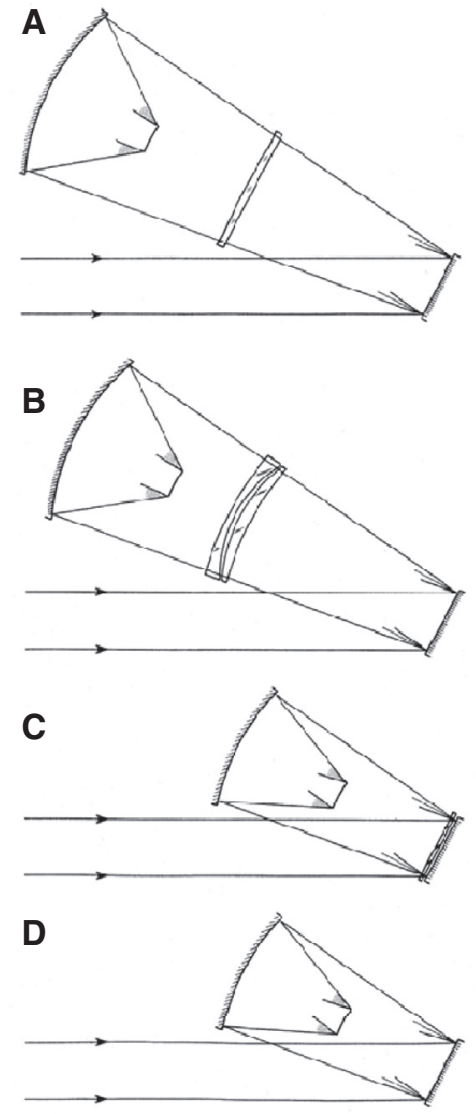

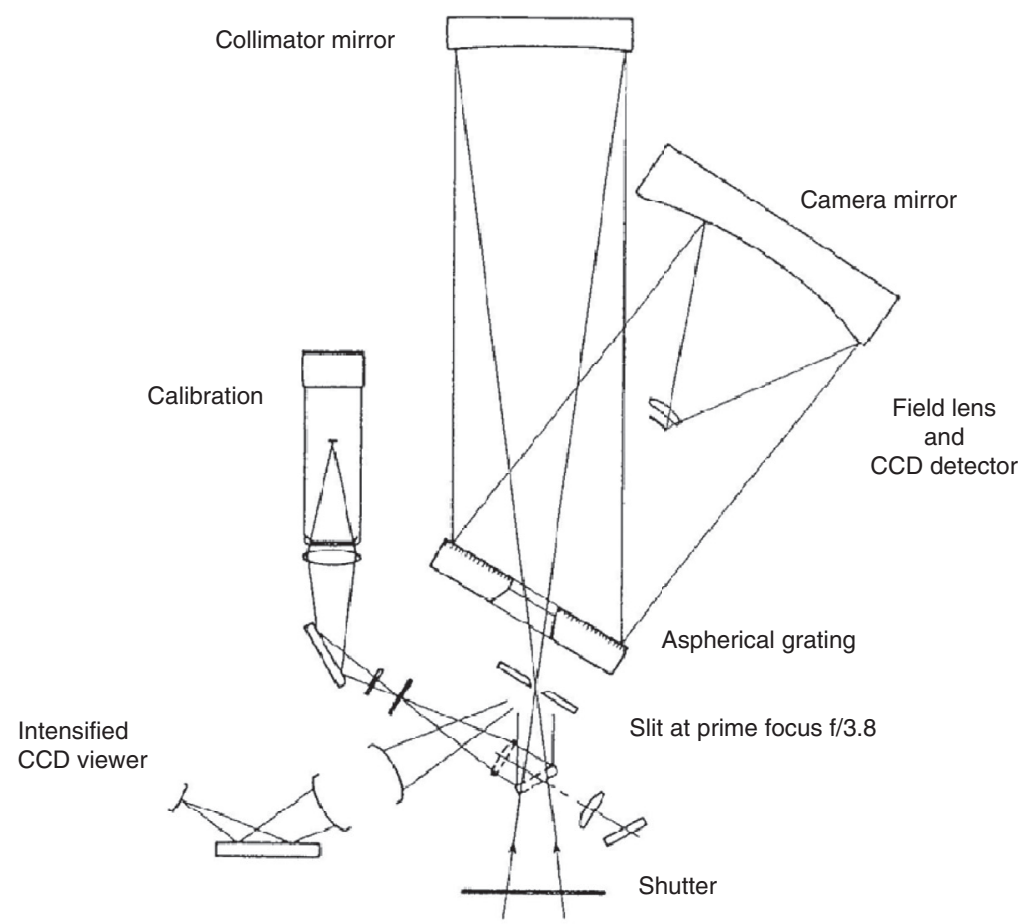

Figure 20 Left: Spectrograph design: comparison of four camera optics with reflective gratings, the same $f$ ratios and pupil at the grating. Design (A) uses an aspheric refractive plate and plane grating. Design (B) is with a null-power doublet lens with spherical surfaces and a plane grating. Design (C) is with a twice-through corrector plate at the plane grating. Design (D) with an aspheric grating is very compact and then drastically minimizes the asphericity to achieve. Right: Optical design of the aspherized grating UV Prime Focus Spectrograph (CFHT). The grating and camera optics make a centered system [1]. 

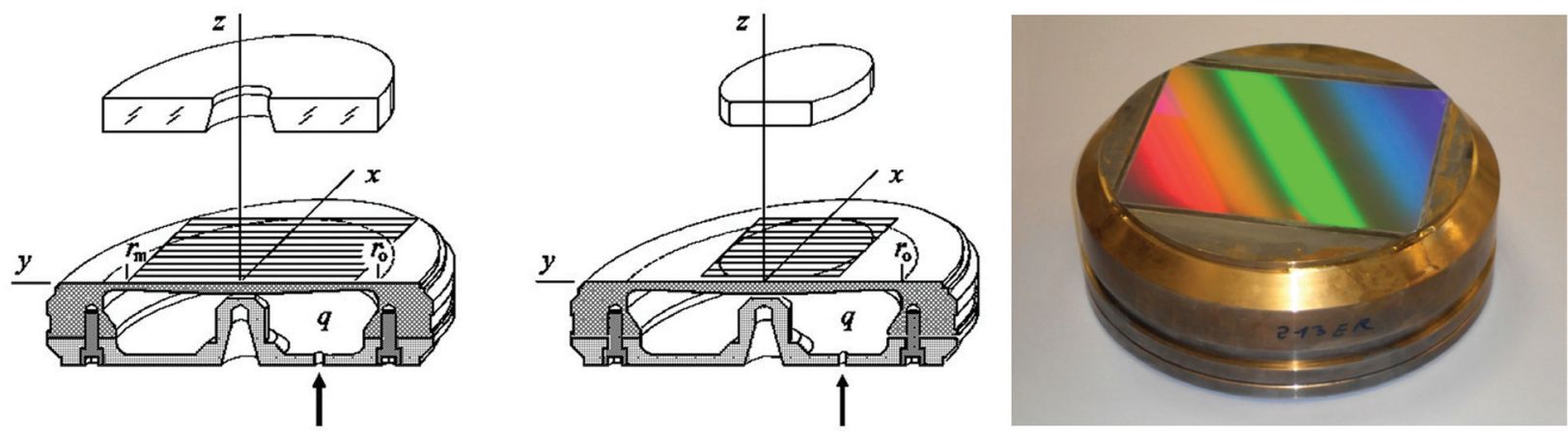

Figure 21 Left: Deformable submasters of quasi-constant thickness distribution (q-CTD) for on-axis - center - and off-axis grating aspherizations by double replicas. Right: View of an intermediate grating replica on an active submaster (LOOM).
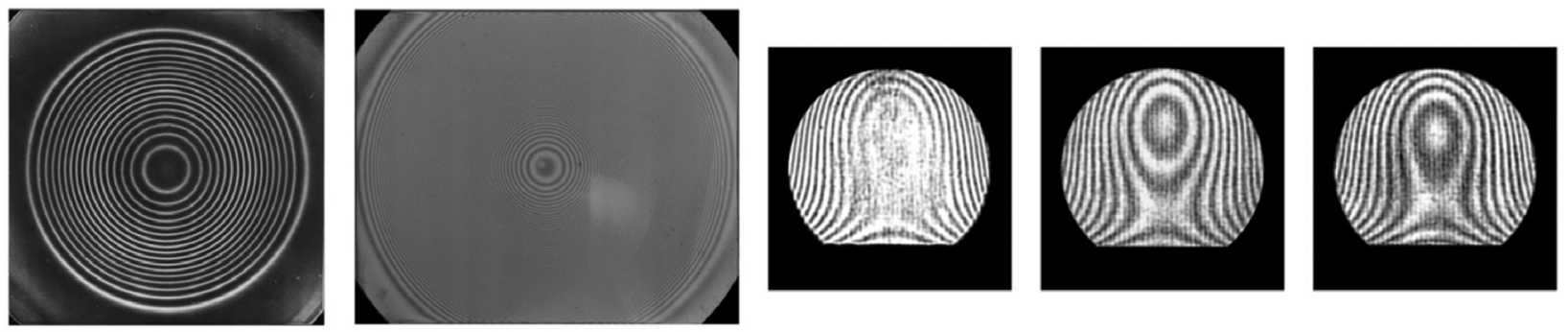

Figure 22 He-Ne fringes with respect to a flat of on- and off-axis reflective grating replicas aspherized on Zerodur substrates. Left: Grating$2,600 \mathrm{l} / \mathrm{mm}, 84 \times 84 \mathrm{~mm}$, of the $\mathrm{f} / 2.8 \mathrm{Marly}-\mathrm{I}$ and $-\mathrm{II}$ spectrographs at OHP and Purple Mountain Observatory. Center: One the five gratings, $1200 \mathrm{l} / \mathrm{mm}, 102-128 \mathrm{~mm}$, of $\mathrm{f} / 2.3$ CARELEC faint object spectrograph at the 2-m telescope of OHP. Right: Off-axis mirror and two gratings, 300 and $600 \mathrm{l} / \mathrm{mm}, 50 \times 60 \mathrm{~mm}$ of the $\mathrm{f} / 5$ ISARD faint object imager-spectrograph at the 2-m telescope at Pic du Midi (LOOM).

\subsubsection{Aspherization of a thin shell secondary mirror}

The hybrid class (HTD) has allowed stress figuring for the hyperbolization of a secondary mirror, $1.2 \mathrm{~m}$ in diameter, as a thin shell adaptive secondary mirror for one of the ESO-VLT 8-m units (Figure 23). The process was proposed and developed by Hugot et al. $[1,11]$.
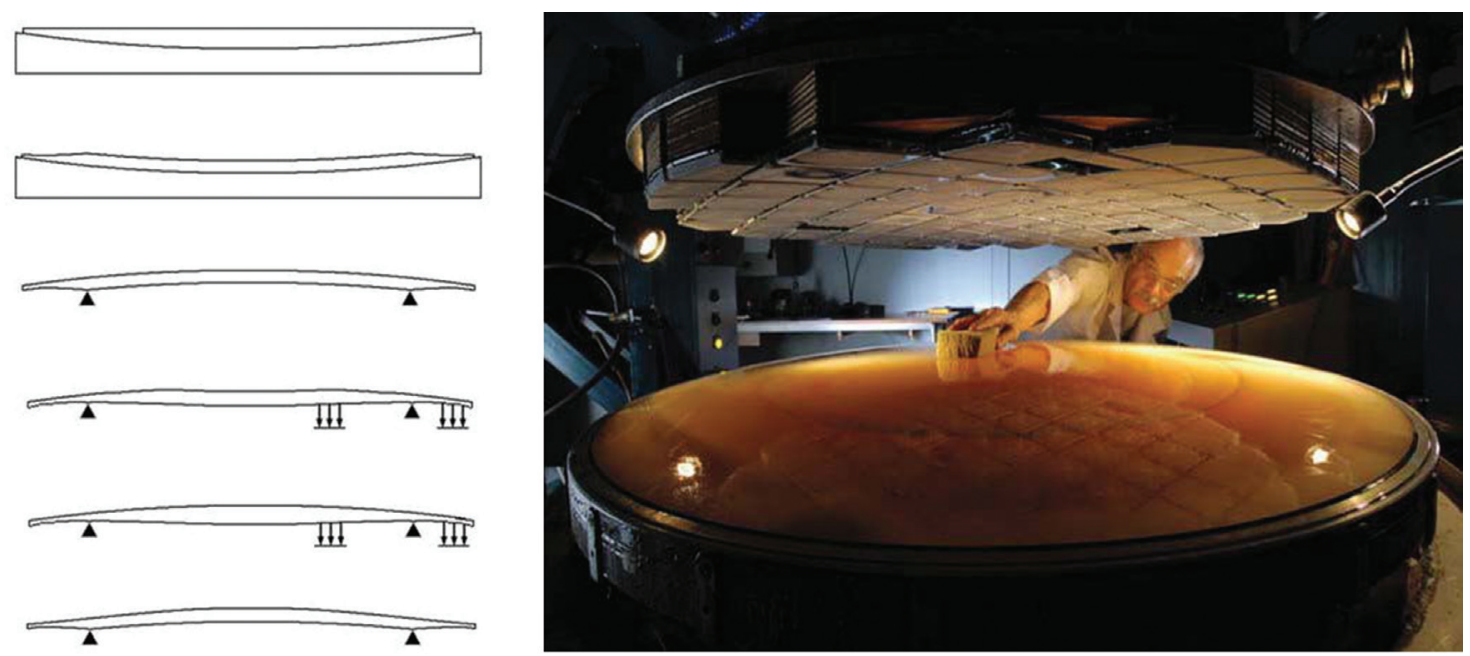

Figure 23 Left: Thin shell aspherization process for the adaptive version of the 1.2-m Cassegrain mirror of the ESO-VLT. Top to bottom: The first three stages for obtaining the thickness geometry of the mirror by diamond turning. Stage 4 shows the loading by partial vacuum over two concentric zones. Stage 5 is the spherical grinding and polishing during the stressing. Stage 6 showed that a smooth aspherical surface was obtained after elastic relaxation of the mirror. Right: View of the thin shell mirror after aspherization and polishing at LOOM. 


\subsection{Primary coma mode $z=A_{31} r^{3} \cos \theta$}

Analysis with the circular thin plate theory shows that primary coma, Coma3, can be generated from either, in the CTD class, by use of a bending moment whose distribution along the substrate contour is of the form $\cos \theta$ or, in the VTD class, by a tulip form on which a net shearing force at the contour varies as $\cos \theta$ in reaction to a bending moment at the substrate center (Figure 24). An easily practicable configuration is a tulip form where a one-directional bridge is built-in at the central zone, and opposite forces are applied to the bridge ends on a simply supported outer cylinder. A hybrid form (HTD) is an equivalent alternative solution [1] (Figure 24).

Applications were developed for obtaining a low dispersion coma-corrected transmission grating. This design was used for slitless spectroscopy with filters in a convergent beam at the $\mathrm{f} / 8$ Cassegrain focus of CFHT
(Figure 25). A plane grating of $75 \mathrm{l} / \mathrm{mm}$ was first deposited on a CTD deformable submaster (Figure 26) and then replicated during stressing onto a transmission BK7 substrate [1].

\subsection{Primary astigmatism mode $z=A_{22} r^{2} \cos 2 \theta$}

The primary astigmatism mode, Astm3, can be generated either from the CTD class or the VTD class. The flexure to be achieved is a saddle-like surface, i.e., with opposite curvatures in the main orthogonal directions. In the CTD class, a basic solution is with bending moments distributed in $\cos 2 \theta$ along the circular contour. With the VTD class, the basic solution is a cycloid-like thickness on which a net shearing force of the form $\cos 2 \theta$ is applied along the mirror contour (Figure 27). Practicable configurations were developed at LOOM by use of either four
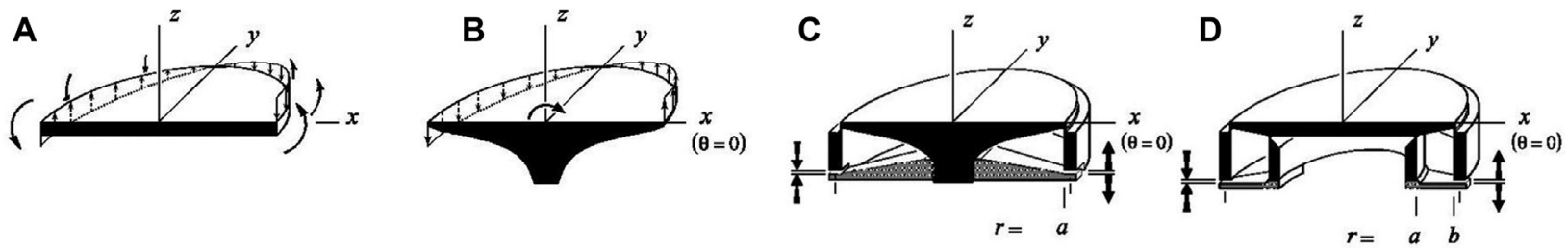

Figure 24 Configurations generating a Coma3 aberration mode. (A) Theoretical configuration of the CTD class applying a bending moment uniformly distributed all along its contour. (B) Theoretical configuration in the VTD class is easily obtained from a perimeter-prismatic forces reacting to a central torque. (C) Practicable configuration in the VTD class derived from the previous one and using two opposite force sets. (D) Practicable configuration in the HTD class using two opposite force sets.

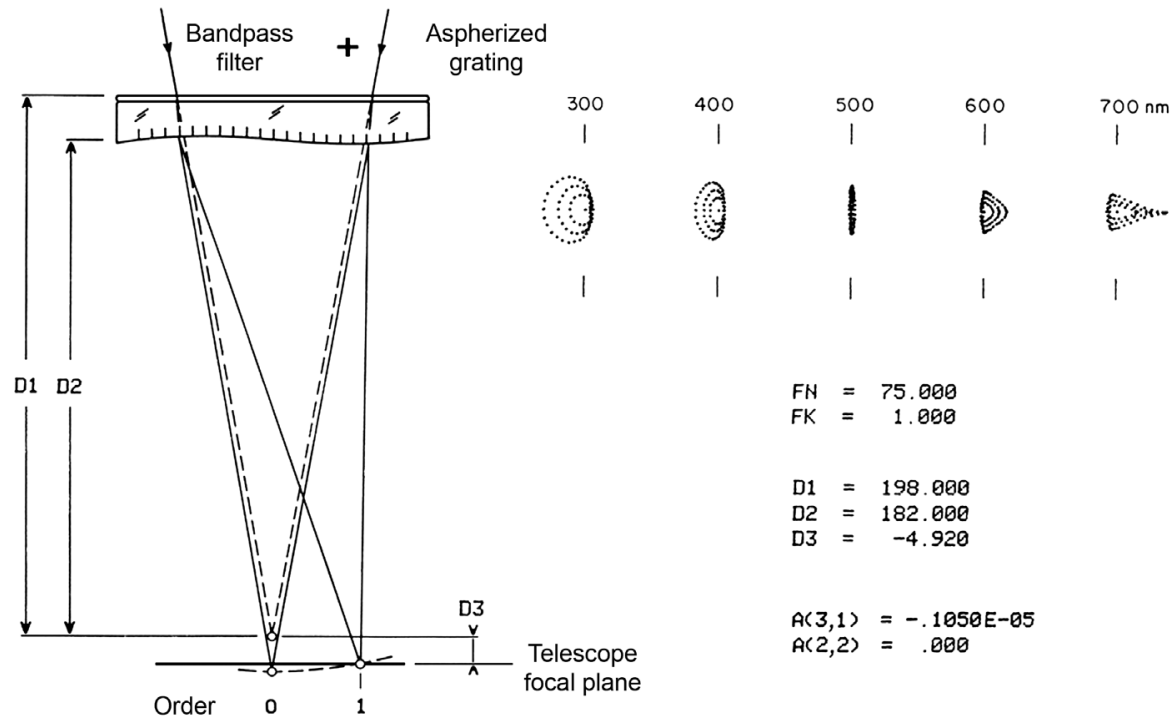

Figure 25 Optical design for objective- or slitless-spectroscopy with Coma3-corrected transmission gratings located in front of the telescope focus. The grating parameters are $F_{\mathrm{N}}=75 \mathrm{~g} / \mathrm{mm}$ and diffraction order $F_{\mathrm{K}}=1$. The coefficients for aspherization of the grating are $A(3,1)=-0.105 \times 10^{-5}$ for Coma3 and $A(2,2)=0$ for $A s t m 3$. The Coma3 deformation over $100 \mathrm{~mm}$ diameter of the active matrix is $\pm 131 \mu \mathrm{m}$ peak-to-valley. 

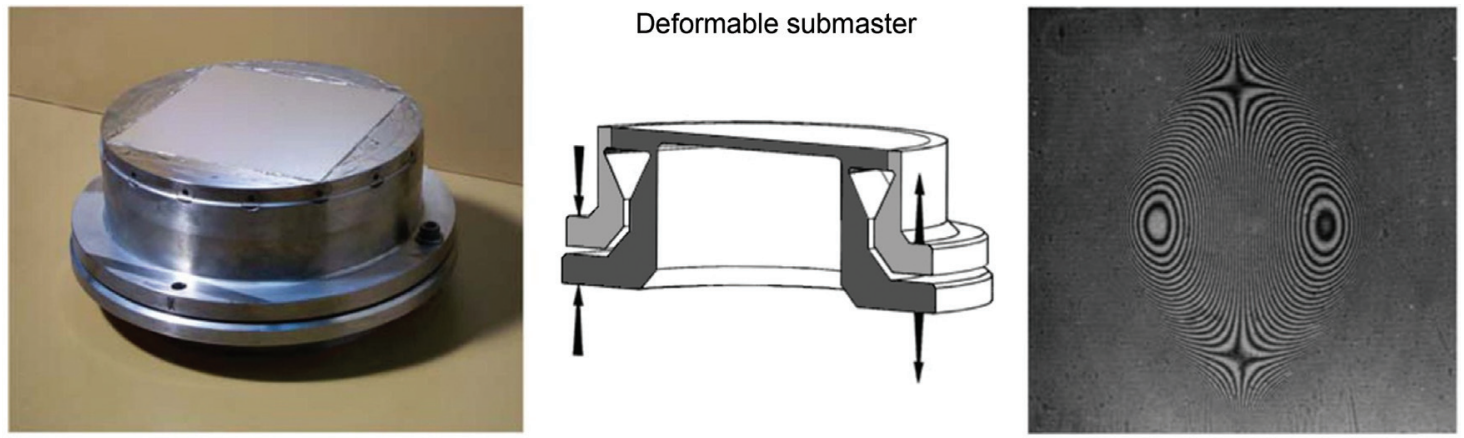

Figure 26 Left and center: View and cut of a stainless steel active matrix for obtaining Coma3-correcting gratings in the CTD class with only two opposite force sets. Right: He-Ne interferogram with respect to a plane surface. Such transmission gratings were used in the convergent beam in front of the Cassegrain focus $f / 8$ of the Canada France Hawaii Telescope (LOOM, Hyperfine Corp. and CFHT).
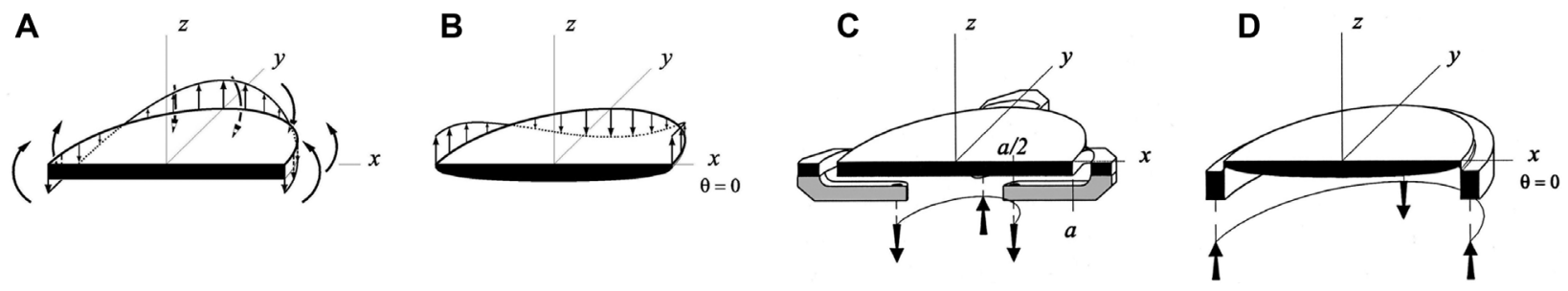

Figure 27 Four elasticity designs generating the Astm3 aberration mode. (A) Theoretical configuration in CTD class requires both perimeter-modulated bending moments and net shearing forces. (B) Theoretical configuration in VTD class mirrors of thicknesses $t / t_{0}=$ constant and $t / t_{0}=\left(1-\rho^{2}\right)^{1 / 3}$. This only requires a perimeter-modulated net shearing force. (C) Practicable solution in CTD class using four bridges and inner arms with two sets of opposite forces. (D) Practicable solution in VTD class using a thin cylindrical collar simply supporting a more rigid outer ring.

outer bending bridges for CTDs or opposite force pairs on a simply supported outer ring of VTDs [1].

Applications were developed for the optical concept of the CDS and UVCS single-surface spectrographs in extreme ultraviolet of the Soho Mission - still continuing solar observations at the Lagrangian point L1 in 2013. These designs by Huber et al. [12] use toroid reflective gratings, which provide two stigmatic points in the dispersed field of the spectra (Figure 28). The process for obtaining the gratings uses a first replication of a spherical grating onto a deformable submaster [1]. Stainless steel submasters, designed in the VTD class, were designed with the cycloid-like form and four azimuthal bridges (Figure 29). A second stage uses a four-force stressing for the final replication on rigid Zerodur substrates.

Other applications have been found useful for the off-axis optics of the planet finder coronagraph SPHERE, a second generation of ESO-VLT instrumentation. Three mirrors differing in size were designed by Hugot et al. [13] as CTD vase form mirrors where the outer rings have
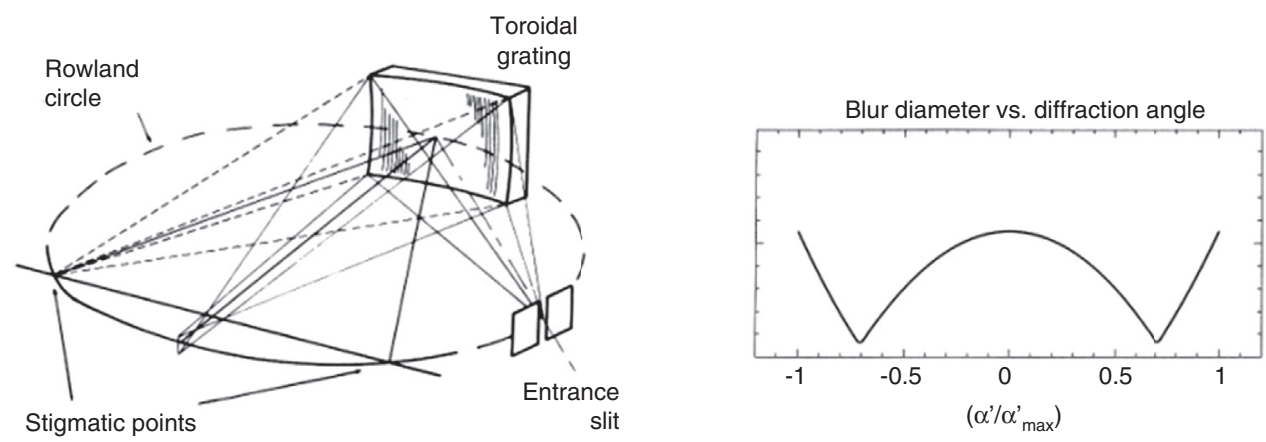

Figure 28 Single surface CDS and UVCS spectrographs of the ESA NASA Soho Mission. The optical design with a toroidal grating provides two stigmatic points (ETH-Zurich). 

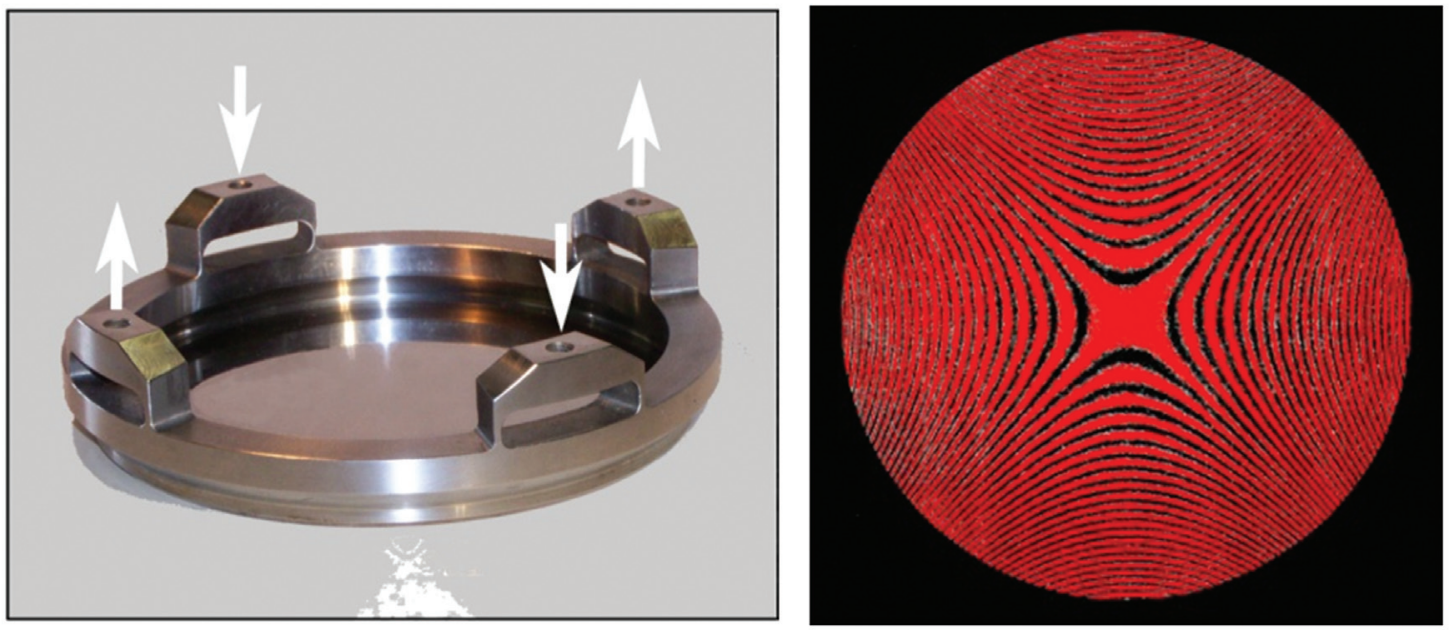

Figure 29 Left: Cycloid-like design in VTD class, i.e., $t / t_{0}=\left(1-\rho^{2}\right)^{1 / 3}$, of a 14-cm deformable matrix generating an Astm3 mode from two opposite force pairs. The one-piece design in stainless steel AISI420 is made of the active mirror with simply supported boundary at its contour realized by a thin cylindrical collar itself linked to a thicker ring including four bridges where each force is applied. This provides an accurate angular modulation in $\cos 2 \theta$. Right: He-Ne fringes of a first replica grating on an active matrix before second replications on Zerodur substrates. The process provided all the toroid gratings of the Soho Mission (LOOM, ETH-Zurich, Bach Research Corp.-Boulder).

azimuthal modulated axial thicknesses in between the four bending forces (Figure 30). Results show that all mirrors have a deviation error with respect to the theoretical toroid shape smaller than $15 \mathrm{~nm}$ rms.

\section{Aspherization of axisymmetric mirrors with fast f-ratios: stress figuring and in situ stressing}

If the mirror curvature is faster than, say, $\mathrm{f} / 3$, then active optics aspherization requires taking into account the stresses induced at the middle surface of the substrate. The shallow shell theory takes these stresses into account for the axisymmetric case (Figure 31) and allows analytically solving the problem of determining the radial thickness distribution for generating the required asphericity - e.g., a conicoid or a spheroid optical shape - from a spherical figure bent by uniform loading. This theory accounts for the strong dependence of the flexure with respect to edge boundary.

The three basic configurations studied are a vase form - which implicitly contains a meniscus form by removing the outer ring - and a closed form (Figure 32). The use of the shallow shell theory on $N$ successive ring segments linked together allows us to determine, via iteration process, the radial thickness distribution set $\left\{t_{n}\right\}$ of the mirror [1].
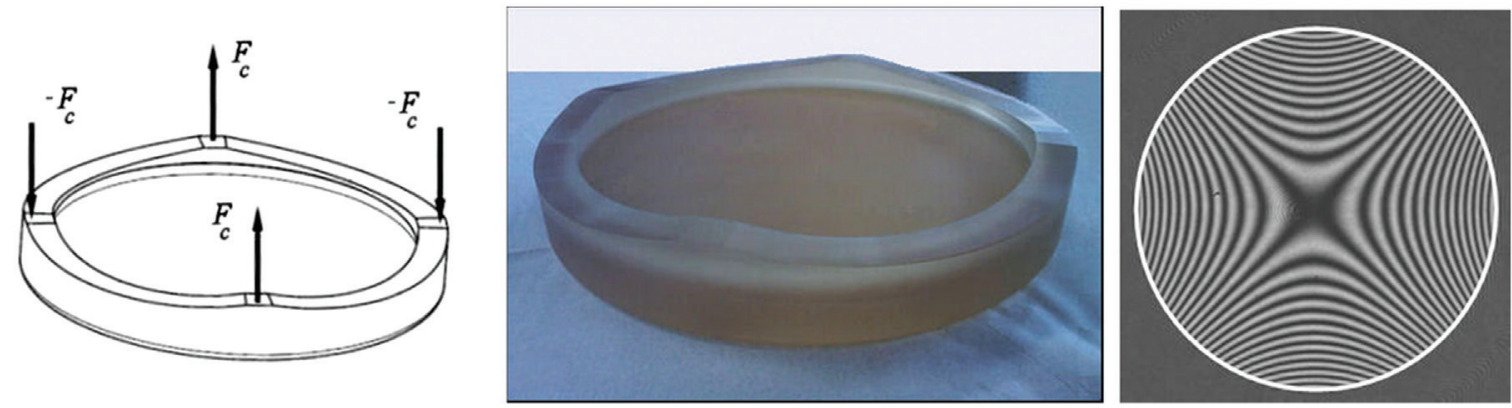

Figure 30 Left and center: Elasticity design in CTD class, i.e., $t / t_{0}=$ constant, of a $40-\mathrm{cm}$ mirror in Zerodur vitroceram bent by four forces during stress figuring. Its contour realizes a built-in boundary to a ring whose axial thickness $t_{R}(\theta)$ shows four azimuth thickness narrowings, $t_{\mathrm{R}-\mathrm{Max}} / t_{\mathrm{R}-\mathrm{Min}}=1.16$, to ensure an accurate modulation in $\cos 2 \theta$. Right: He-Ne interferogram with respect to a mean curvature sphere of one of the three Astm3-correcting mirrors made for SPHERE. This instrument is a next-generation planet finder of the ESO-VLT (LOOM, Thales SESO Corp.). 


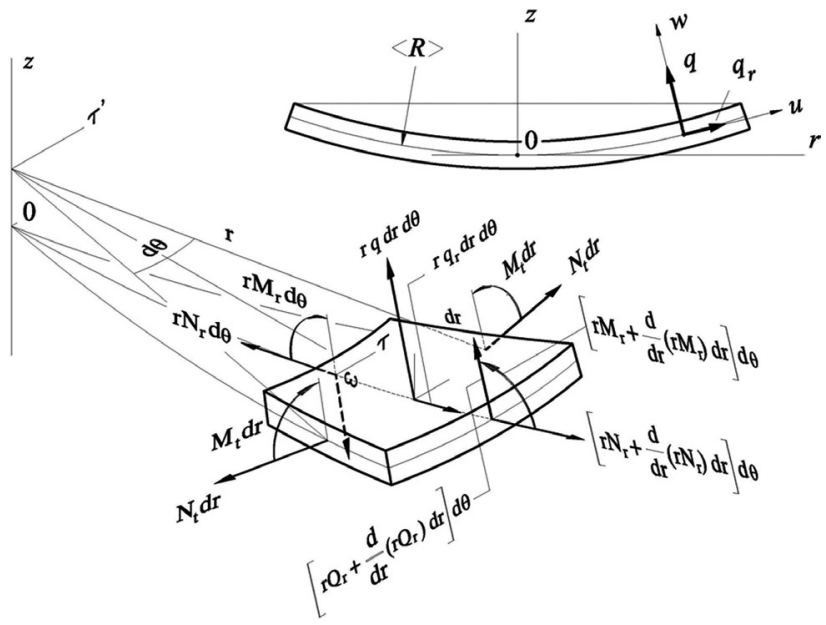

Figure 31 Shallow shell theory and finite element in cylindrical coordinates. Equilibrium of the elementary forces. These include forces lying at the middle surface of the shell.

\subsection{In situ parabolization of concave mirrors}

The parabolization of a concave mirror by stress figuring is a very difficult task because the uniform load to be exerted is not a partial vacuum - which would naturally flatten the mirror against the table of the machine - but an inner air pressure, which then requires use of a continuous and accurate reaction system along the mirror contour. Depicting this tremendous difficulty for large telescope mirrors, a more natural alternative for smaller-size mirrors, say up to $4 \mathrm{~m}$ in diameter, is to make a spherical figuring without stress and then to practice in situ partial vacuum at the telescope by closing the rear side of the mirror.

Experience gained with a holed mirror aspherized by in situ stressing led us to retain a vase shell design with an optimized radial thickness distribution $\left\{t_{n}\right\}$ for an $\mathrm{f} / 1.75,20-\mathrm{cm}$ clear aperture paraboloid mirror in Zerodur. The back side of the outer ring is given a folded L-shape to increase the perimeter rigidity without overweighting (Figure 33). The central hole is closed with a paste on a soft sliding tube. Another inner tube applies, via a conical spring, a shearing force, which provides an equivalent force distribution to that of the plain mirror [1].

\subsection{Mirror hyperbolizations of a modified- Rumsey three-reflection telescope}

Starting from the three-mirror anastigmatic telescope proposed by Rumsey in 1969 [14] and an extensive study by Rakish on three- and four-mirror telescopes [15], a very interesting optical system for wide-field sky surveys in astronomy - typically $1^{\circ}$ or $2^{\circ} \mathrm{FoV}$ - is a three reflection modified-Rumsey telescope (Figure 34). This flat field anastigmatic system avoids the use of three- or four-lens correctors of single-primary-mirror systems, or two-lens correctors for flat-fielded Ritchey-Chrétien systems, and then is completely free from chromatic aberrations except that it is due to the spectral band filter and the detector
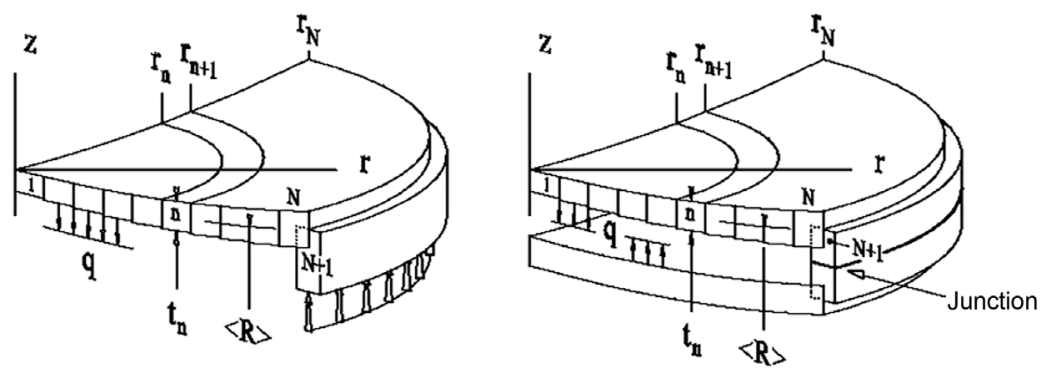

Figure 32 Geometries of a vase form mirror and closed form mirror for analytical determination of the VTD.
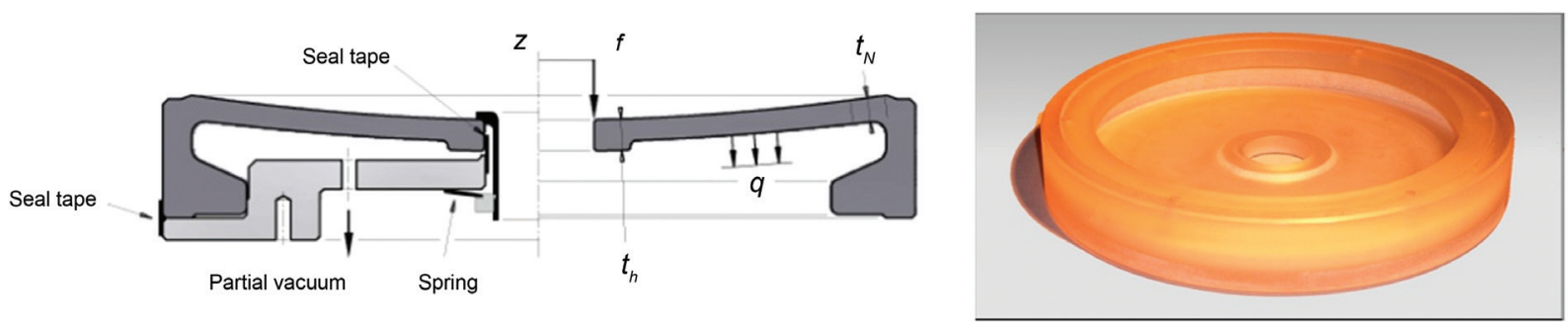

Figure 33 Elasticity design and view of a holed f/1.75 vase form mirror parabolized by in situ stressing via air pressure (LOOM). 

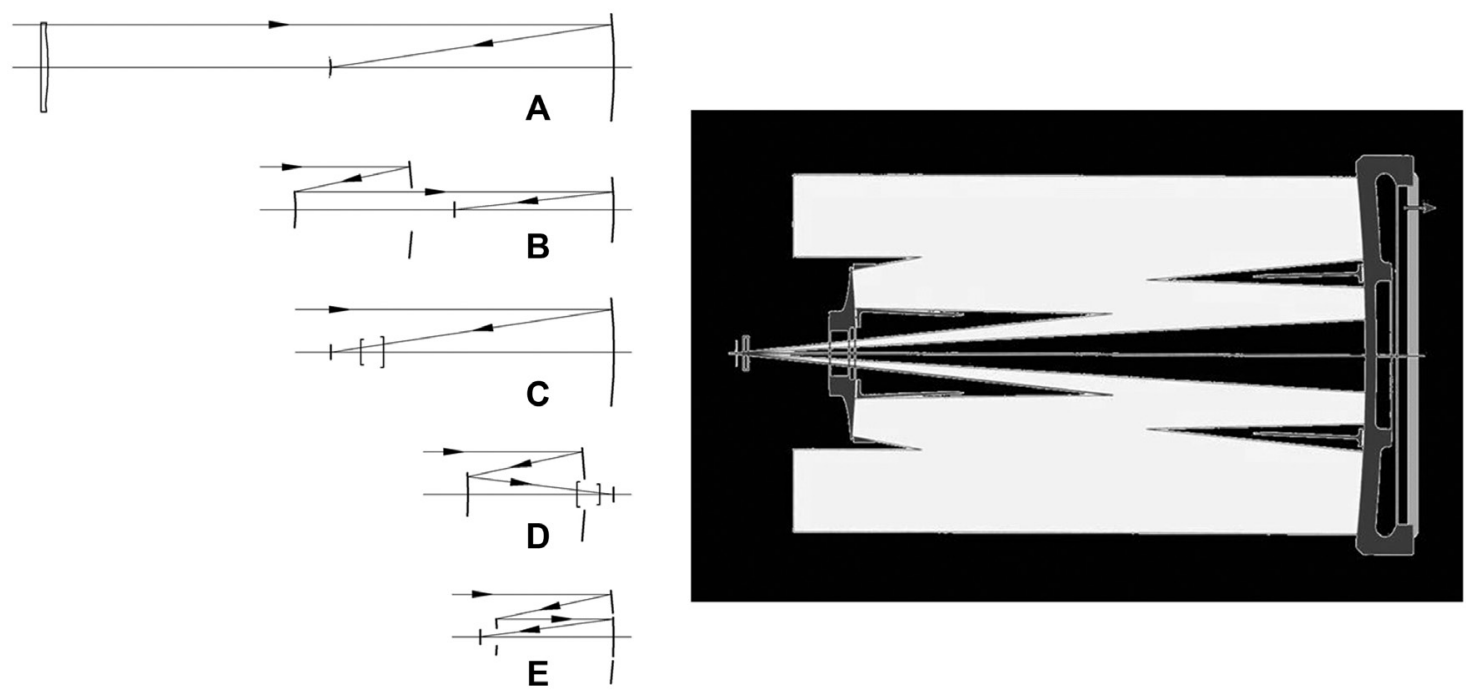

Figure 34 Left: Comparison of five wide-field telescope designs: (A) Catadiotric Schmidt. (B) Mersenne Schmidt. (C) Prime focus with three or four-lens corrector. (D) Cassegrain R-C with two-lens corrector. (E) Modified Rumsey. Right: Three-reflection telescope MINITRUST: modified-Rumsey flat-field anastigmat design (E) with surface, slope, and curvature continuities between M1 and M3 mirrors.

window plate, as for all usual optical systems. In a modified-Rumsey form, elasticity analysis shows that both primary and tertiary mirrors can be simultaneously hyperbolized by in situ stressing at the telescope - by partial vacuum - after spherical figuring with a lap acting over the whole substrate surface of a double-vase form $[1,16]$. This form is made of a vase form included at the central part of a larger vase form.

Two 50-cm aperture, $2^{\circ}$ FoV, modified-Rumsey telescope demonstrators, MiniTrust I and II, were built at LOOM. All three mirrors were given a spherical figure with only two rigid laps. The double vase substrate of M1-M3 mirrors allowed their simultaneous hyperbolization by in situ stressing. Hyperbolization of the tulip form M2 was achieved by stress figuring (Figure 35). Results from a whole-telescope autocollimation test show a deviation error smaller than $280 \mathrm{~nm}$ PtV, i.e., 48-nm RMS for a single-pass wavefront (Figure 36).

\subsection{Aspherization of convex mirrors by stress figuring}

The aspherization of telescope secondary mirrors into a paraboloid or hyperboloid by uniform loading and stress figuring is straightforward. This is because a partial vacuum is applied to the rear face of the mirror, which then naturally flattens it on the table of the figuring machine. A typical value of the uniform load applied by partial vacuum corresponds to $75 \mathrm{kPa}$, i.e., $3 / 4 \mathrm{Atm}$, allowing to make under- or overcorrection for the stress polishing. We developed the parabolization of vase form Cassegrain mirrors made of ultralow expansion titanium-silica (ULE) for $1.5-\mathrm{m}$ afocal telescopes. These mirrors were designed and built for Labeyrie [17] at GI2T-CERGA to provide a beam compression ratio of 20 (Figure 37).

Two $25-\mathrm{cm}$ aperture vase form convex mirrors in Zerodur were hyperbolized by stress figuring. For
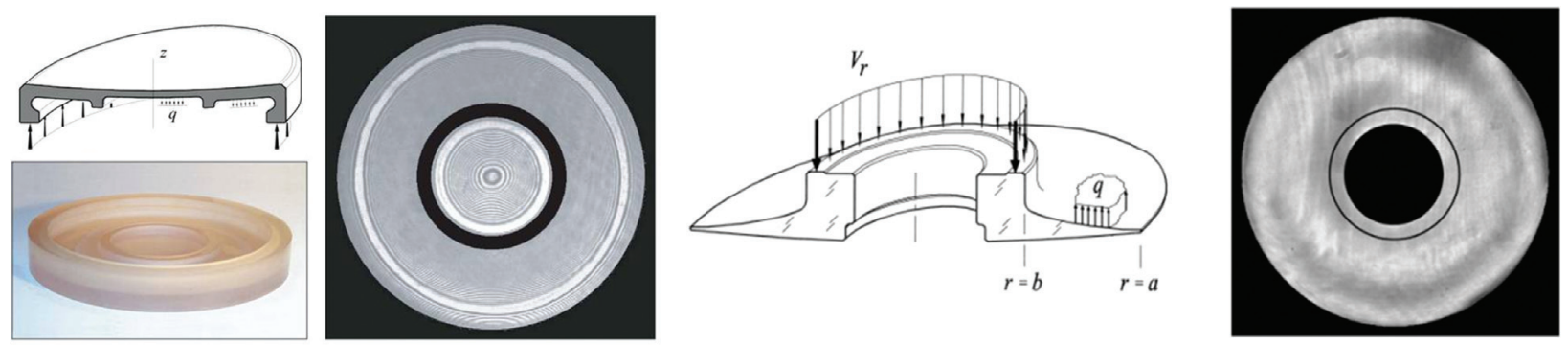

Figure 35 MINITRUST mirrors. Left: Double-vase form M1-M3 mirrors hyperbolized by in situ stressing. Right: Tulip-form M2 mirror hyperbolized by stress figuring (LOOM). 


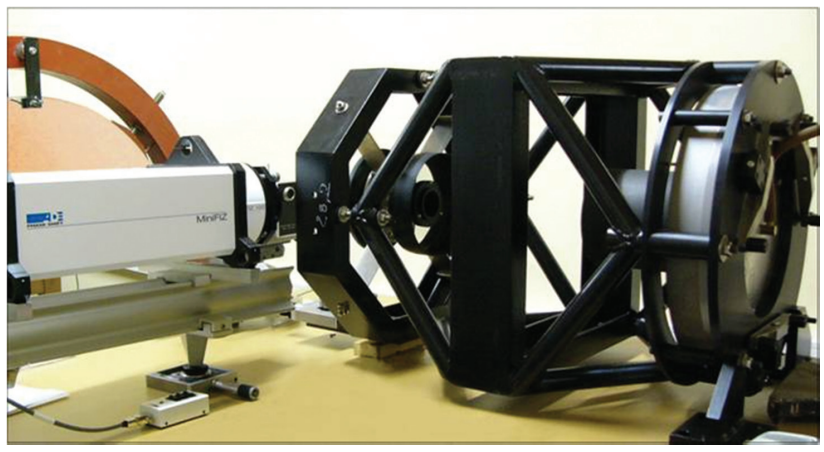

Figure 36 View of MINITRUST during optical testing with wavefront sensor. This used a double pass via a plane reference mirror (LOOM).

compactness, their design used an inner folded outer ring (Figure 38). The thinness of the reflective aperture allows the efficient implementation of a backside cooling system [1]. These are Cassegrain mirrors of the 1-m RC THEMIS Solar Telescope at Tenerife, in the Canary Islands.

\section{Multimode deformable mirrors (MDMs): vase form and meniscus form}

Except for very special cases - such as the Astm 3 and $C v 1$ modes, which can be generated by the same cycloid-like thickness $t / t_{0}=\left(1-\rho^{2}\right)^{1 / 3}$ in the VTD class with very few actuators - the superposition of various optical modes requires an elasticity design, which belongs to the CTD class. Vaseform or meniscus-form multimode deformable mirrors (MDMs) can be bent by forces applied to outer radial arms and by optional uniform load over the whole mirror surface $[1,18]$. For large telescope mirrors, it is preferable to use force actuators uniformly distributed over their rear surface.

\subsection{MDMs with outer arms and Clebsch- Seidel modes}

Because of the similar form of Seidel optical modes and the Clebsch flexural mode that are general solutions of Poisson's bilaplacian equation $\Delta^{2} \Delta^{2} z+q / D=0$, where $q$ is the uniform load and $D$ the constant rigidity, one can show that from the thin plate theory, there exists a common subset of modes similar to Seidel modes. We call them Clebsch-Seidel modes [1].

Denoting a mode as $z=A_{n, m} \cos m \theta$, this subset is generated by terms $m=0$ for $q=$ constant, and $m=n$ and $m=n-2$ for $q=0$. These terms are the Sphe 3 mode and all modes of the two lower diagonal lines $D_{1}, D_{2}$ of the optics triangle matrix modes (Figure 39). Shown interferograms were obtained from a 20-cm MDM with $K=12$ arms for which axial forces $F_{a, k}$ and $F_{c, k}$ are applied to the inner and outer end of arm-k, respectively (Figure 40).
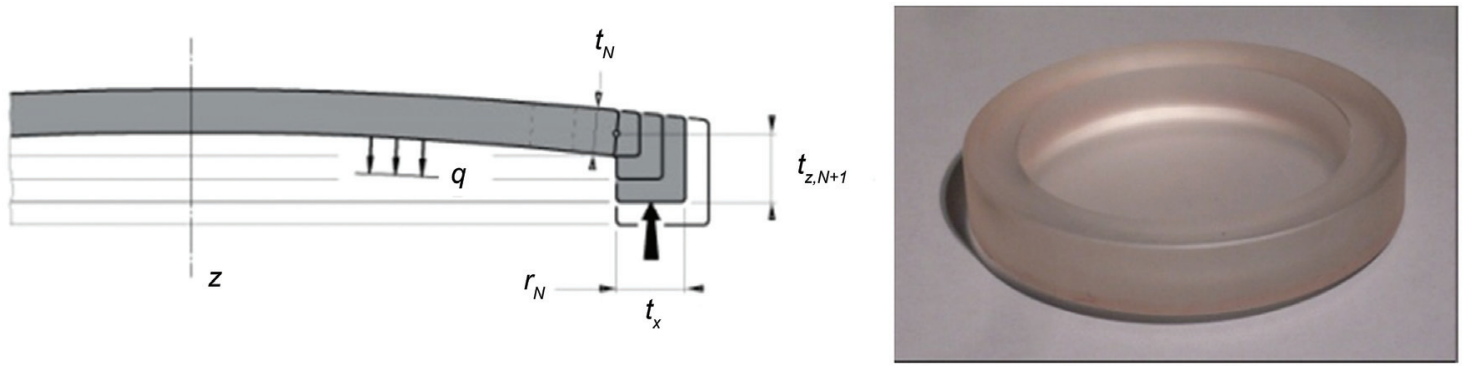

Figure 37 Left: Convex vase form mirrors parabolized by stress figuring. Right: View of a fused-silica ULE mirror for the GI2T (LOOM).
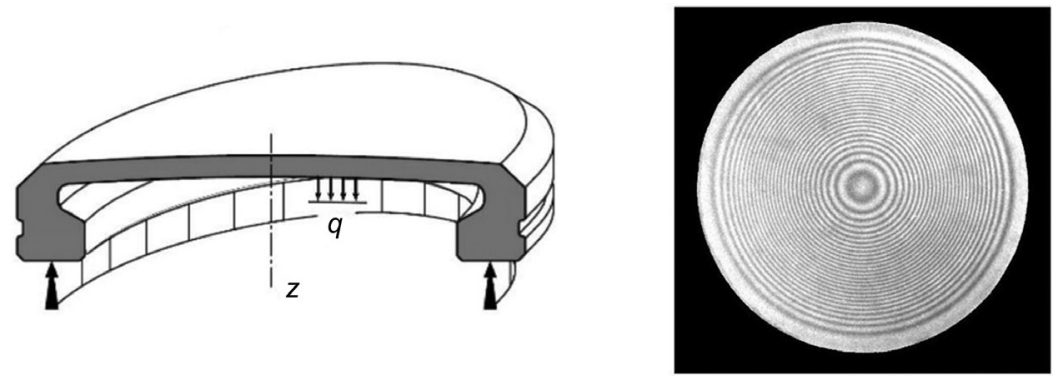

Figure 38 Left: Vase form secondary mirror of the THEMIS Ritchey-Chrétien telescope hyperbolized by stress figuring. Zerodur substrate. Right: He-Ne interferogram with respect to a spherical concave caliber. Clear aperture $25 \mathrm{~cm}$. Mirror surface error $40 \mathrm{~nm}$ peak-to-valley (LOOM). 


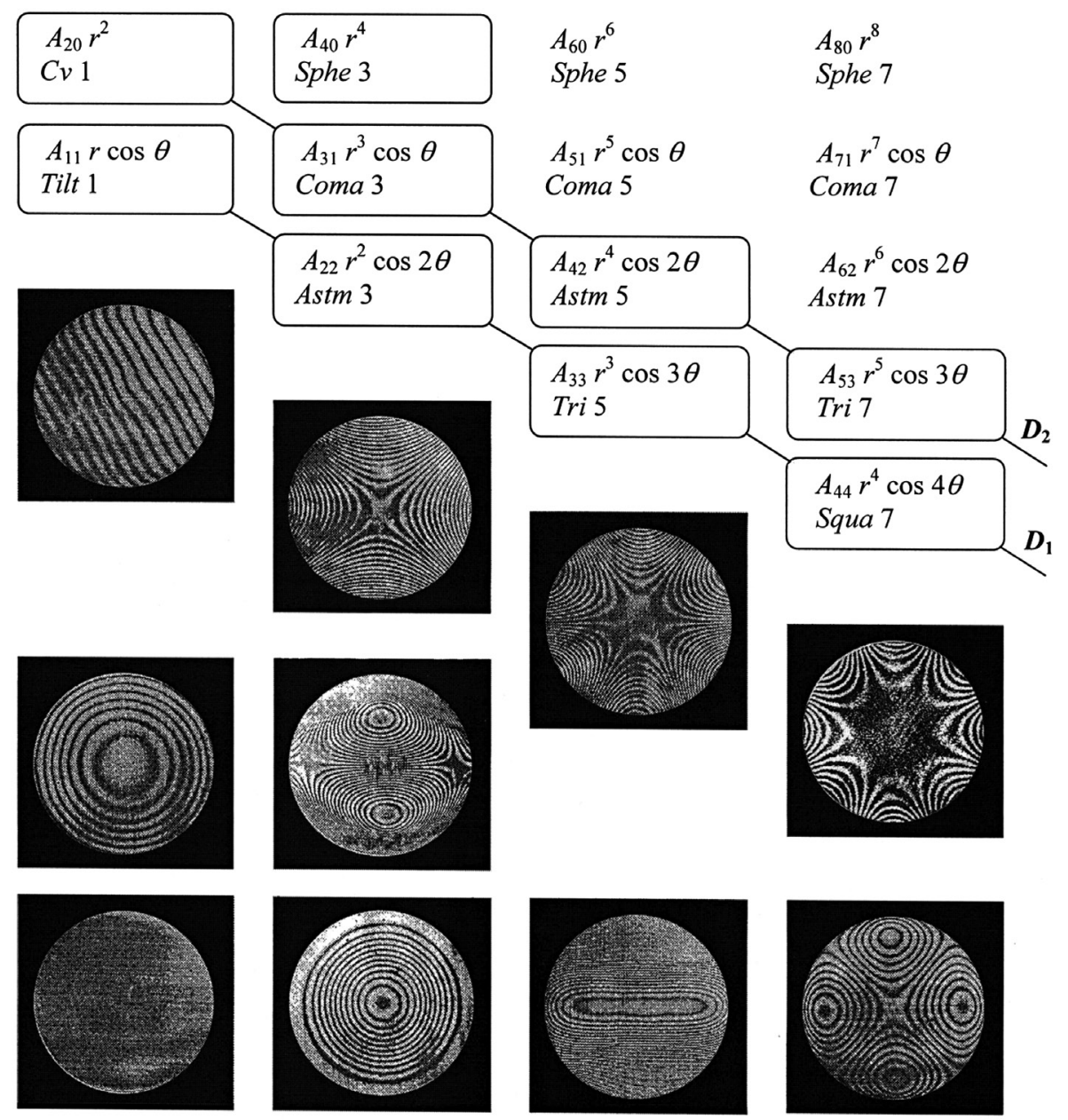

Figure 39 Up: Triangle matrix of Seidel optical modes. The Clebsch-Seidel modes are shown in boxes. Down: Some Clebsh-Seidel modes obtained with a 12-arm MDM (Figure 40). The last row displays the flat MDM at rest on the left and two composite modes on the right (LOOM).
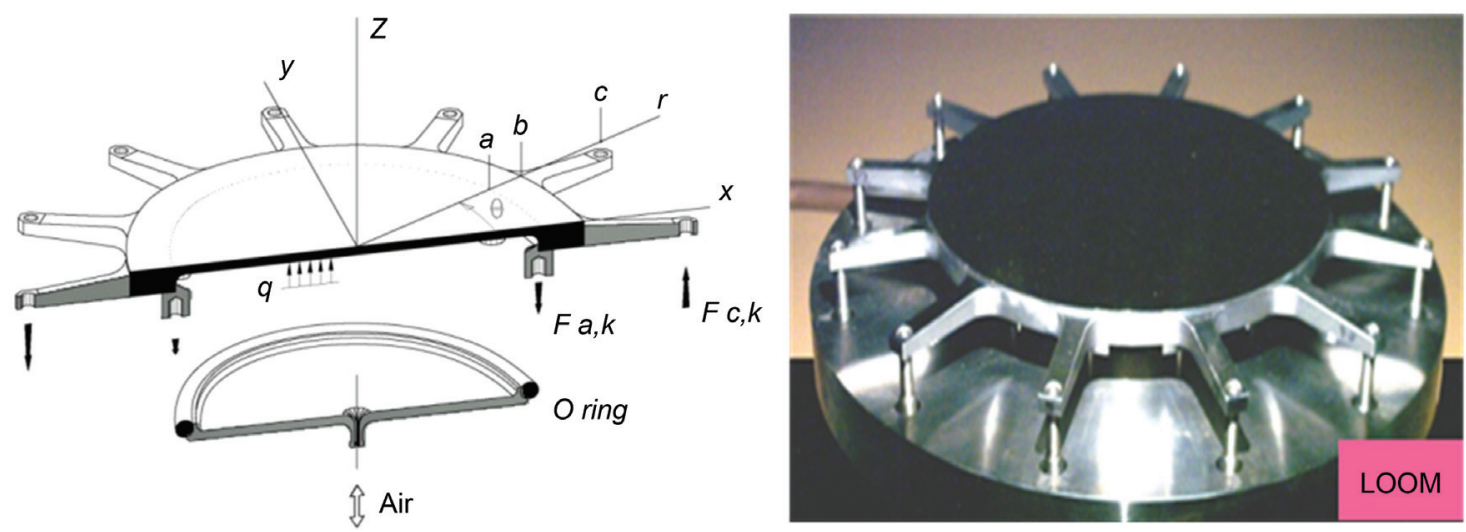

Figure 40 Elasticity design and view of a 12-arm vase form multimode deformable mirror - MDM (LOOM).

\subsection{Degenerate configurations: monomode mirrors}

Generating a single mode with an MDM equipped with $K$ radial arms generally requires use of $2 K$ forces. One can show from thin plate theory that some configurations, called degenerate, require a maximum of $K$ forces only. A family of them is for $m=n$. Experimental results have provided confirmations for $m=n=2$ and $m=n=3$, i.e., Astm3 and Tri5 modes [1, 18]. For instance, a vase form, 
made of quenched stainless steel FeCr13, provided a pure Astm3 mode with four folded arms and four forces only (Figure 41).

\subsection{Meniscus MDMs: Keck telescope segments}

MDMs originated from a theoretical study by Lubliner and Nelson [19] for the segmented 10 m, f/1.7, Keck telescope, the largest optical-infrared telescope. Nelson et al. [20] applied the method to the construction of 36 segments of the primary mirror. The stress figuring aspherization process of 1.8-m segments was applied to circular Zerodur meniscuses. $K=24$ radial arms - acting on the edge through stuck plates - distributed the bending moments and net shearing forces to generate the superposition of the Cv1, Coma3, and Astm3 modes (Figure 42).

\subsection{Meniscus in situ reshaping and telescope alignment control}

With the construction of the 3.5-m ESO-NTT, Wilson et al. [21, 22] pioneered new concepts to achieve the best possible image quality during observations with large telescopes. The thickness of the primary mirror is much thinner than usual, with an aspect-ratio $t / D=1 / 15$, thus allowing in situ reshaping by a set of support actuators. The secondary mirror is actively set up to optimal position, avoiding decenter, and tip-tilt. Wavefront sensors tracking natural stars provide the necessary information for analysis. Closed loop systems provide efficient control drives whatever the position is on the sky. The concept

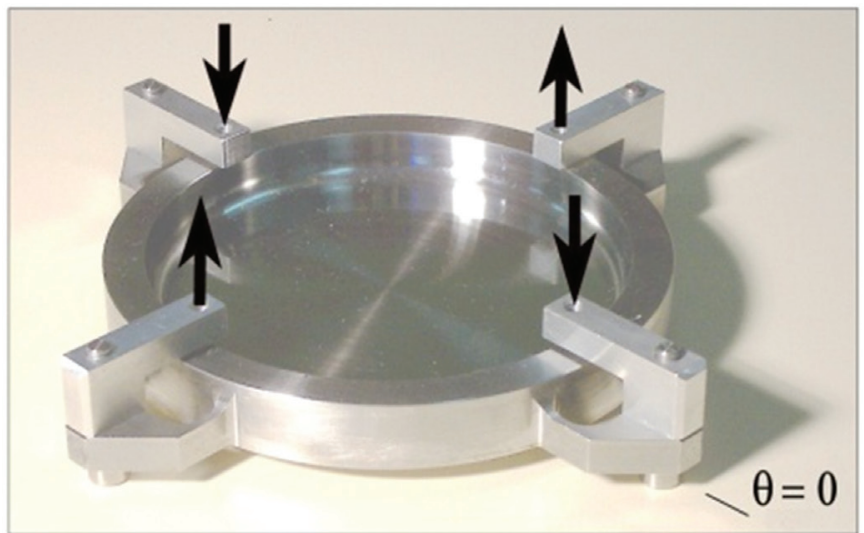

was then applied successfully to the four ESO 8.2-m units, $t / D=1 / 47$, of the ESO-VLT (Figure 43).

\subsection{Meniscus MDM: giant reflective Schmidt LAMOST}

The giant reflective Schmidt LAMOST - also called Guoshoujing telescope - is a meridian telescope dedicated entirely to multi-object spectroscopy. A 4.2-m clear aperture and $5^{\circ}$ field of view give this telescope an unprecedented optical etendue [1]. Four thousand optical fibers are remotely positioned over a focal surface of $1.75 \mathrm{~m}$ in diameter. The fiber outputs feed 16 double spectrographs.

The concept by Wang, Su et al. [23] provides today's highest spectrum-acquiring rate in the world (Figure 44). The active optics in LAMOST is the most extensive development ever carried out, with the 24 flat segments of the primary mirror aspherized in situ as a function of the deviation angle and transit angle from the meridian $\left( \pm 15^{\circ}\right.$ for a 2-h exposure time). The elliptic contour of mirror M1 (Figure 45) was designed for a maximum deviation from the sky corresponding to a declination angle $\delta=+90^{\circ}$. The shape and position of each M1 segment is generated by 37 force actuators and three displacement actuators. The LAMOST active optics systems were conceived and developed by Su et al. [24, 25] and Cui et al. [26, 27].

\subsection{Universal aberration compensator for holographic grating recording - vase form MDMs}

Universal MDM compensators for low- and high-order aberrations have been proposed and developed for

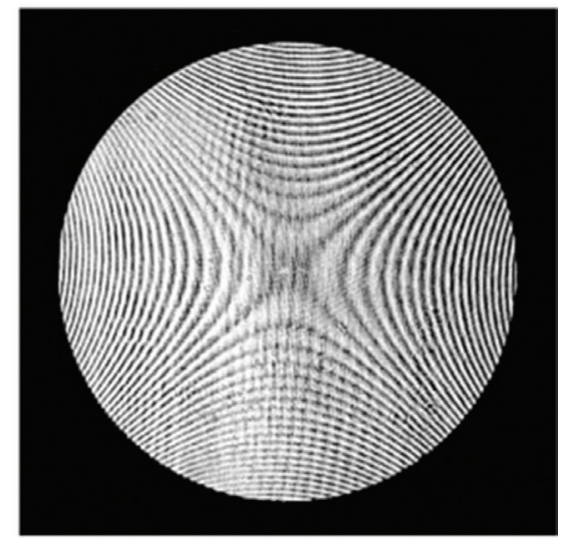

Figure 41 Degenerate vase form CTD quenched stainless steel mirror - Cr13Fe alloy - for $m=n=2$ generating Astm 3 with only four forces instead of eight forces (LOOM). 

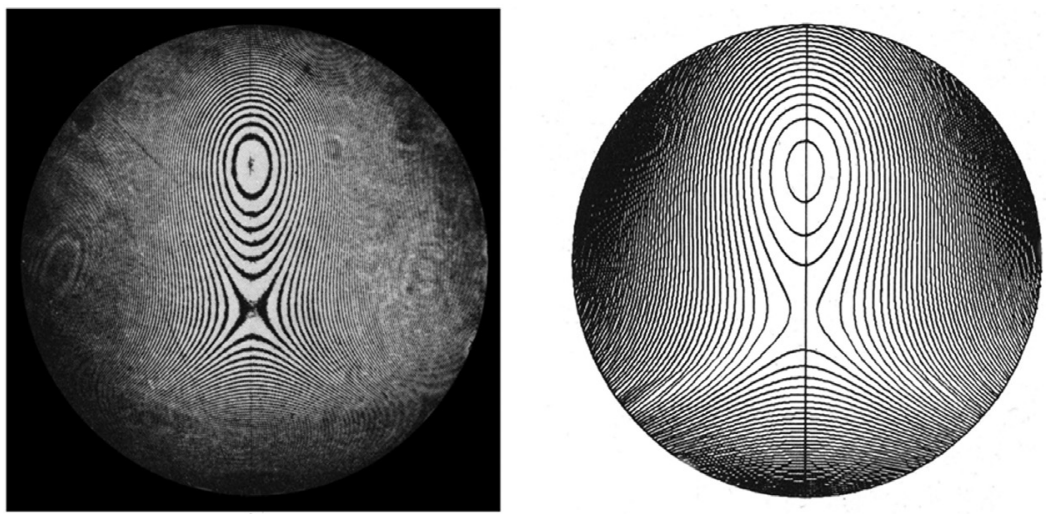

Figure 42 Actual and theoretical shapes of an outermost segment aspherized by stress figuring of the 10-m Keck telescopes (Univ. of California).

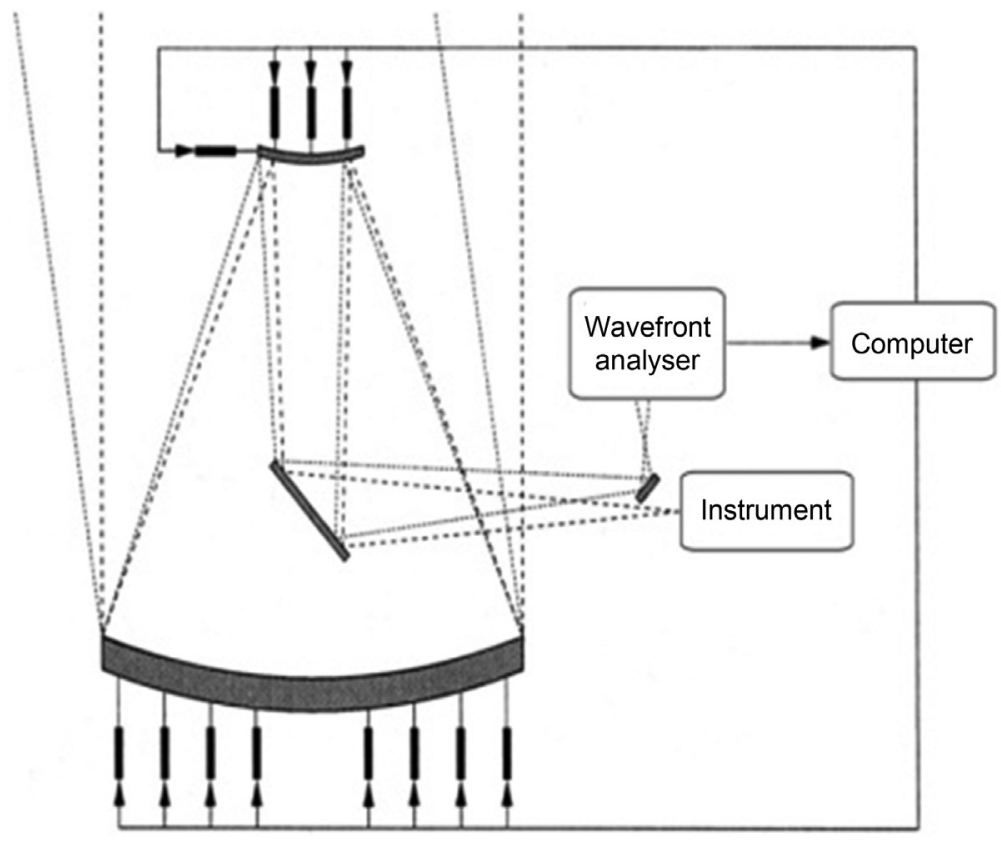

Figure 43 Principle of close-loop control for a two-mirror telescope. The active process includes reshaping of a large meniscus primary mirror and decenter plus tip-tilt of the secondary mirror.
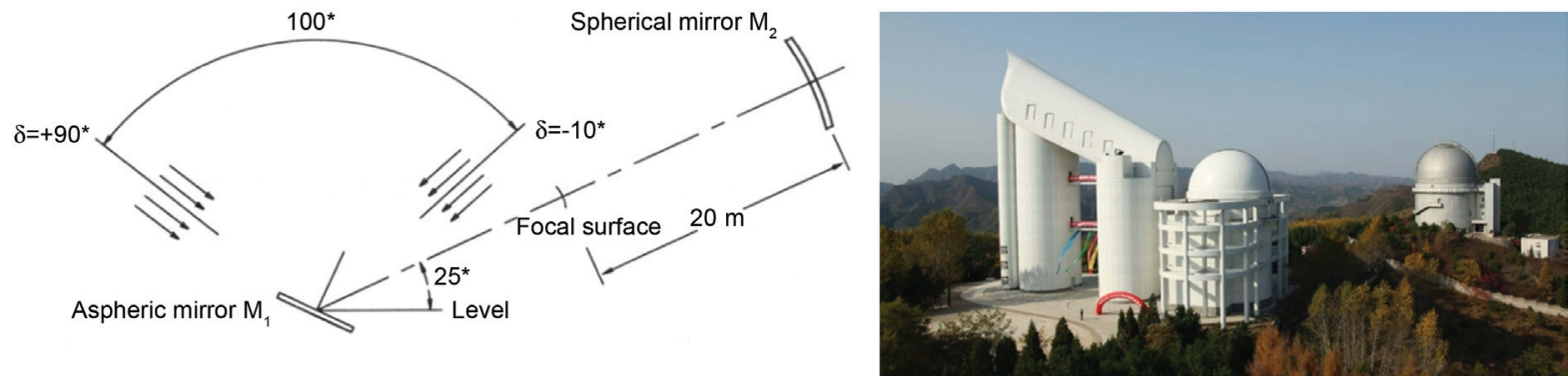

Figure 44 Left: Optical design of giant reflective Schmidt LAMOST. Right: View at Xinglong Station Observatory (NAOC/CAS).

holographic recording of concave gratings. Adopting a vase form, this was applied to the Cosmic Origins Spectrograph - COS - of the Hubble Space Telescope. Depicting of nominal results (Figures 46 and 47), our proposal was not finalized for COS. However, this would have led to a gain of 1.5 in magnitude and four in spectral resolution. 

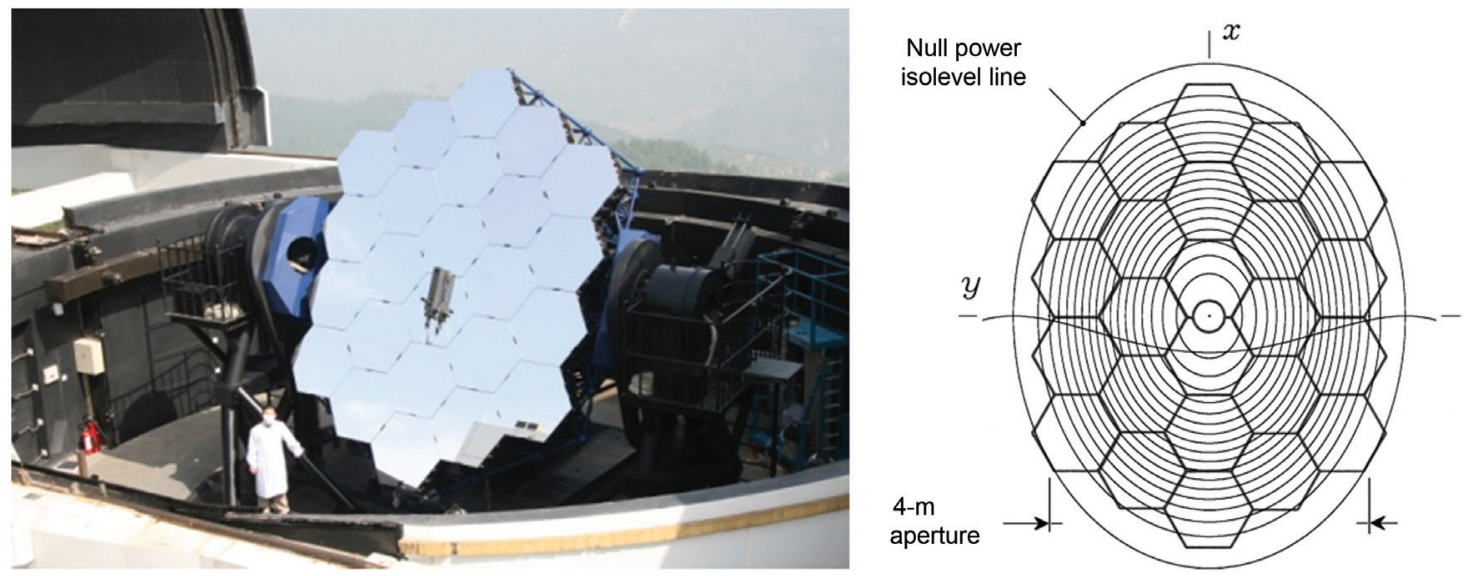

Figure 45 Left: View of LAMOST active optics primary mirror. Right: The ellipticity of iso-level lines of the primary mirror M1 to be actively aspherized is a function of the incidence angle from the sky.
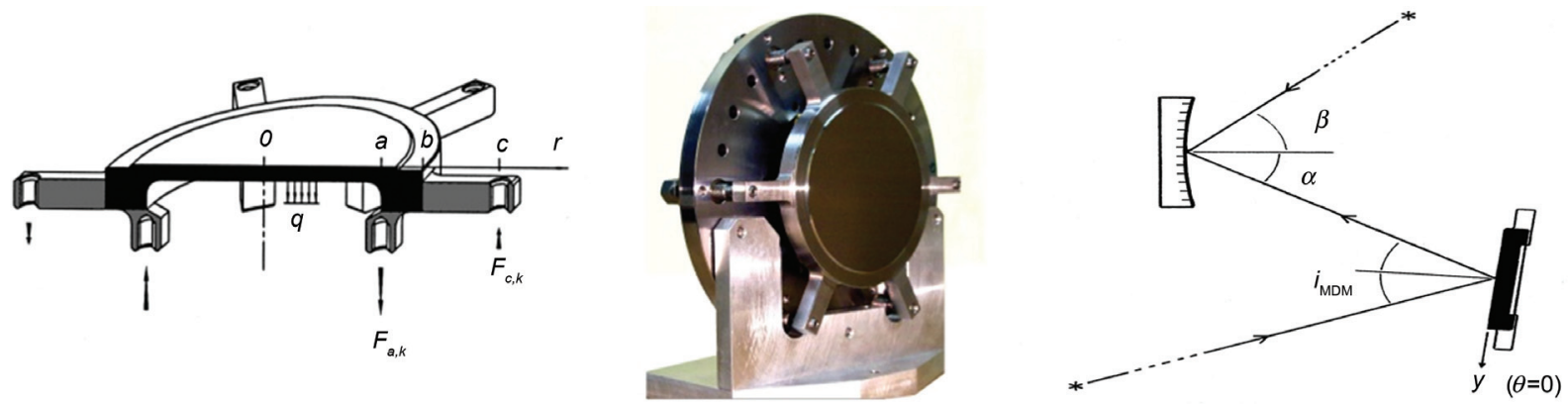

Figure 46 Left: Elasticity design and view of a six-arm vase form MDM. Center: View of the MDM during stressing. Right: Optical mounting for the holographic recording of aberration corrected gratings (LOOM).
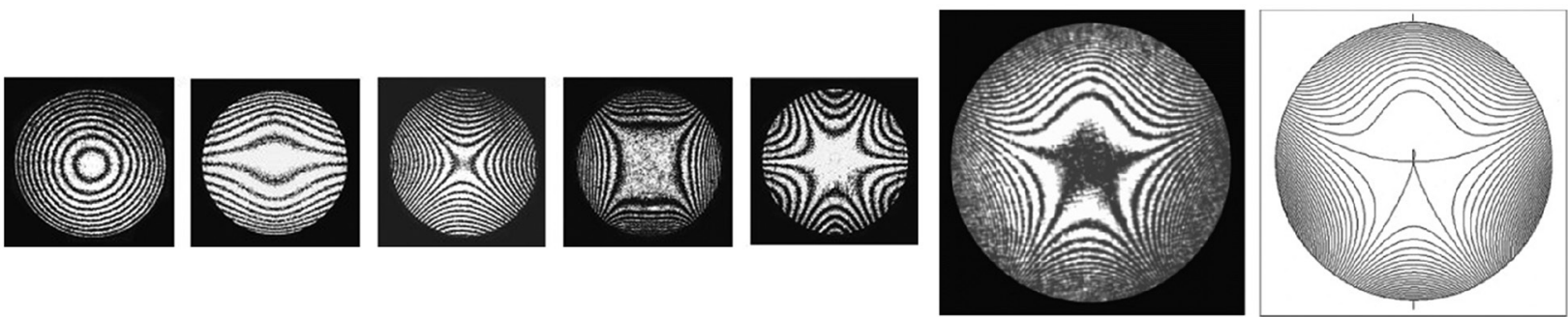

Figure 47 Left: CV1, Coma3, Astm3, Tri5, and Squa7 modes (cf. triangle matrix in Figure 40) generated by a six-arm MDM. Right: Mode superposition: experimental and theoretical shape for the COS/HST holographic grating recording (LOOM).

The recording compensator was a six-arm vase form MDM in stainless steel quenched alloy [1, 28, 29].

\subsection{Aspherization process for the E-ELT segments}

We are presently developing a new industrialization procedure based on the vase form MDM concept [1] for aspherizing the outermost M1 segments of the European
Extremely Large Telescope (E-ELT) project by the European Southern Observatory. This 39-m design is a five-mirror train, which includes a pupil transfer for ground layer corrections on adaptive mirror M4 and field stabilization mirror M5 (Figure 48).

Mirror M1 will be made of $\sim 900$ hexagonal segments, $1.35 \mathrm{~m}$ in diagonal, which form an f/0.9 slightly elongated ellipsoid - i.e., with a conic constant slightly lower than unity. The aspherization process was developed by Ferrari et al. [30], Laslandes et al. [31], and Hugot and Floriot. 

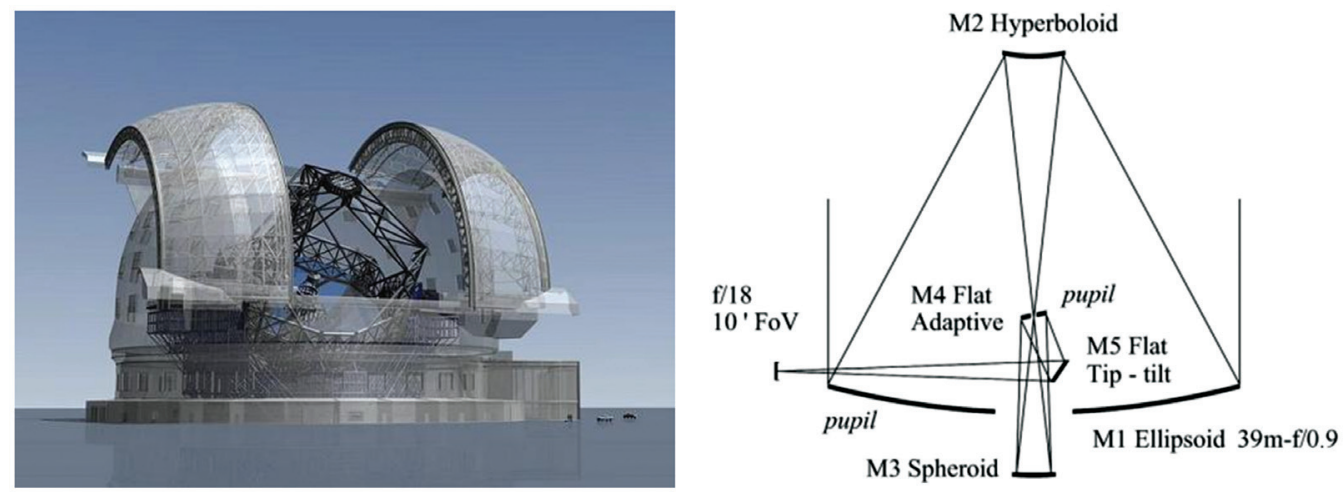

Figure 48 Artist's view of the E-ELT Project is a 39-m five-mirror design including pupil transfer on the adaptive mirror M4 and tip-tilt mirror M5 (ESO).

It will use a 12-arm MDM stressing harness [1] where a Zerodur segment is linked to an active ring during aspherization (Figure 49).

\section{Minimum number of actuators and shape control of large telescope mirrors}

Considering large ground-based telescopes, the main mirrors supported by pads in their cells are submitted to two flexural modes caused by the effect of gravity: i) the axial deformation mode (ADM) which is maximum at the zenith, and ii) the lateral deformation mode (LDM) when off-zenith. Concerning the ADM, the required pad density supporting the mirror per unit surface area, to maintain a correct optical figure, is well determined as a function of any peak-to-valley tolerance criterion, mirror material and thickness, spacing of the pads, and the size of the pads (Figure 50) [1].

\subsection{Large monolithic mirrors}

For large monolithic mirrors, such as the existing VLT $8.2 \mathrm{~m}$-diameter and $175 \mathrm{~mm}$-thick thin meniscuses in vitroceram, the peak-to-valley flexural sag of the ADM was partly reduced by using, during the grinding and polishing, a pad geometry quite similar to that of the final telescope cell. The pads supporting the mirror were made of 150 axial force-actuators distributed over 6 concentric circles, thus providing a quasi-uniform pad density of $2.9 / \mathrm{m}^{2}$. Not taking into account the fact that the pad-print of the ADM is diminished by $\sim 50 \%$ during grinding and polishing would lead to adopt a greater pad density of $3.8 / \mathrm{m}^{2}$ for $70 \mathrm{~nm}$ ptv pad-print flexures at the mirror surface ([1], pad density law given by eq. 8.25). The particularly good concept of the VLT mirror lateral force actuators, which minimize the LDM, allows the small cylinder-like deviation modes to be fully corrected by the axial force actuators with the adopted pad density of $2.9 / \mathrm{m}^{2}$ whatever the zenith distance.

If one notices that the ratio of the pad radius over the distance between each pad is small (ratio $\sim 1 / 6$ for the VLT
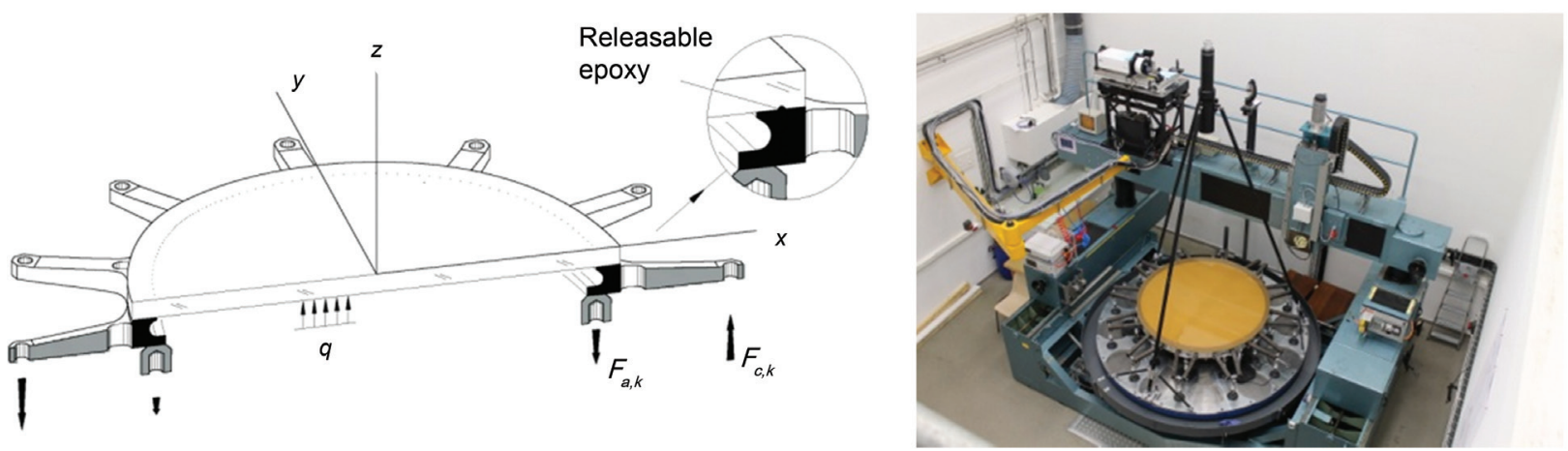

Figure 49 Vase form MDM assembly with active deformable harness for the active optics aspherization of E-ELT segments. View of the 2.5-m platform at LOOM. 

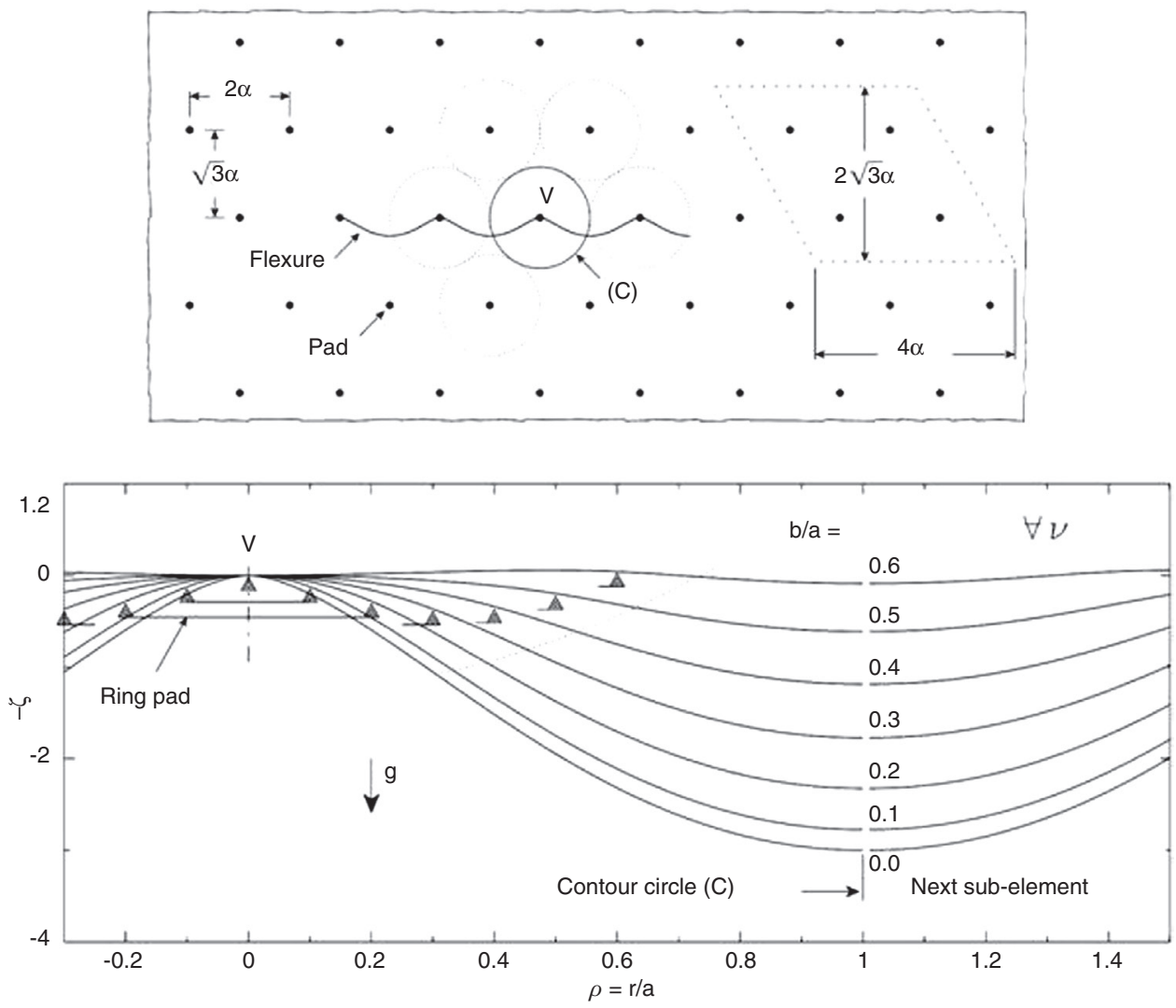

Figure 50 Up: Infinitely large mirror of constant thickness axially supported by equally spaced pads distributed over a 3-fold symmetry. Inter-space $2 a$ between two consecutive pads. Down: Flexure dependence of a mirror sub-element area $(C)$ as a function of ring diameter $2 b$ of the pad support whatever the Poisson's ratio $v$, for $1 / 5<v<1 / 3$. For $b / a=0$, the ring diameter of the pad reduces to a single point. When $\mathrm{b} / \mathrm{a} \simeq 0.6$, the flexural sag is at a minimum.

mirror), then for the monolithic meniscus the selected density of actuators and the pad density adopted to minimize the ADM and LDM are identical.

\subsection{Segments of large telescope mirrors}

The segments of current extremely large telescope projects, such as the TMT and E-ELT of 30 and $39 \mathrm{~m}$ in aperture respectively, will be mounted on tripod frames providing active optics corrections of the tip-tilt and piston modes by displacements of three pad actuators of the tripod. The segments are hexagons in zero expansion vitroceram, 1.4-1.5 $\mathrm{m}$ in diagonal length, and $50 \mathrm{~mm}$ thickness.

The E-ELT project is a three mirror anastigmat including addition of a pupil transfer between a pair of flat mirrors, adaptive mirror $\mathrm{M}_{4}$ and field stabilization mirror $\mathrm{M}_{5}$, which fold the beams along the elevation axis to the Nasmyth foci (Delabre [32], Vernet et al. [33]). The ADM of a segment is minimized by an axial support system called a whiffletree - a superimposition of pivot triangles - mounted on the tripod frames. A hollow cylindrical imprint made at the back center of the segment allows the lateral support system to provide, via a membrane plate link, negligible sag of the LDM. Maintaining the accurate optical surface of a segment mainly resumes to a simple passive design with the whiffletrees. Three ninepoint two-level whiffletrees provide a support system of 27 axial pads for the segment which is mounted on 3 axial displacement actuators. Thus, the density of the pads is 9 times that of the actuators. The $39 \mathrm{~m}$ mirror $\mathrm{M}_{1}$ is made of 798 segments which leads to a pad density of $36 / \mathrm{m}^{2}$ and an actuator density of $3.9 / \mathrm{m}^{2}$.

For the E-ELT project, one notices that the actuator density of 3.9-units $/ \mathrm{m}^{2}$ does not really differ from that of the VLT monolithic mirror. Because of the lower thickness of a segment ( $5 \mathrm{~cm}$ instead of $17.5 \mathrm{~cm}$ for the VLT), and then by the flexure due to gravity increasing as the second power of the thickness ratio, i.e., a ratio of $\sim 12$, the pad density is substantially higher than that of the VLT.

At present optomechanical investigations of the E-ELT segment supports by Cayrel [34] lead to the introduction of a pair of actuators applying orthogonal moments at 9 


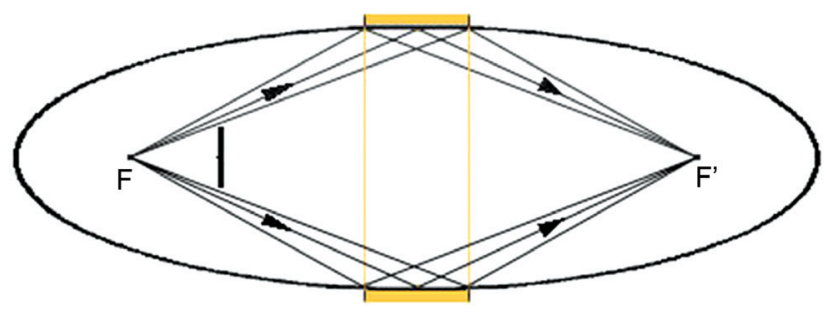

Figure 51 Conjugate foci of an elongated ellipsoid used in grazing incidence.

pivots of the whiffletrees. These 18 actuators should allow the generation of non-uniform force distributions for slight corrections of $C v 1, A s t m 3$, and possibly Tri5 modes. However this complexity would lead to 16758 actuators for $\mathrm{M}_{1}$ instead of 2394 for the piston and tip-tilt modes only.

\section{X-ray optics and active optics}

The extremely short wavelengths of X-rays require the fabrication of mirrors showing super-smooth surfaces. This is without doubt one of the most important features of $\mathrm{X}$-ray optics because slope discontinuities due to ripple errors entail absorption and scattering effects, which may severely degrade the performance. One usually allows a surface roughness not exceeding 2-3 Â. For this reason, active optics figuring with rigid laps and in situ active optics would greatly improve the performance of aspheric tubular mirrors. This technique has not been much used up to now except for long stripe mirrors in synchrotron instrumentations.

\subsection{Grazing incidence: $\mathrm{X}$-ray relay mirrors}

For example, starting from a toroid tube - i.e., where the meridian sections are segments of a circle - the elasticity theory of tubular shells allows obtaining meridian sections of a stigmatic elongated ellipsoid. This tubular shape is required to build accurate relay mirrors used in synchrotron facilities for X-ray instrumentation tests (Figure 51).

Let us consider a rotational symmetry tube, around its $\chi$-axis, bent by a uniform load $q$ applied outside or inside all along the surface of the tube, one may notice that generally the radial deformation - extension or compression - is always of the same sign, i.e., of monotonic sign, all along $\chi$. If one assumes now a monotonic sign extension

Parabolic flexure
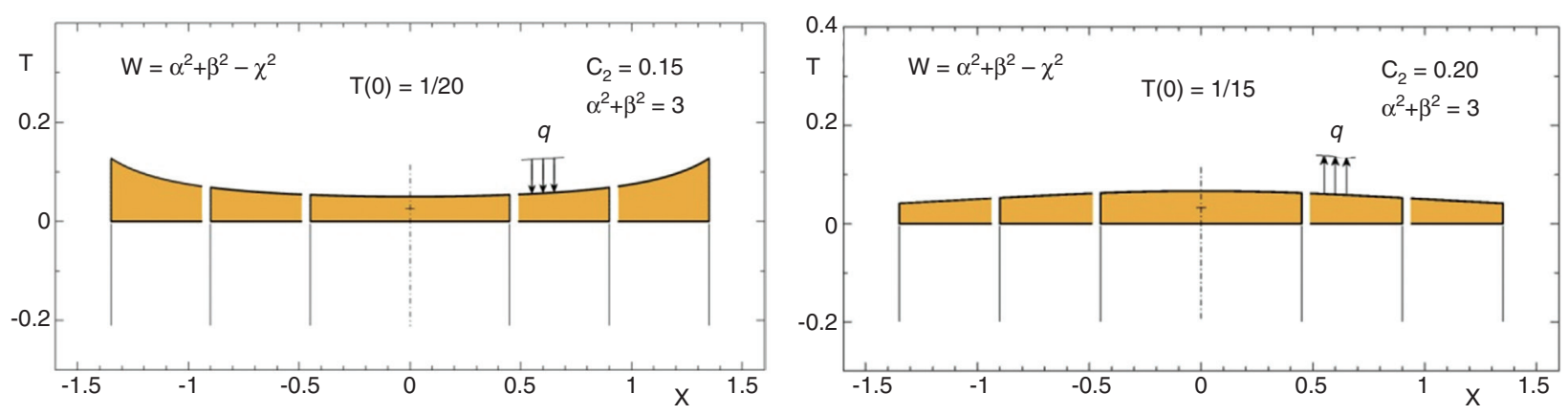

Fourth degree flexure
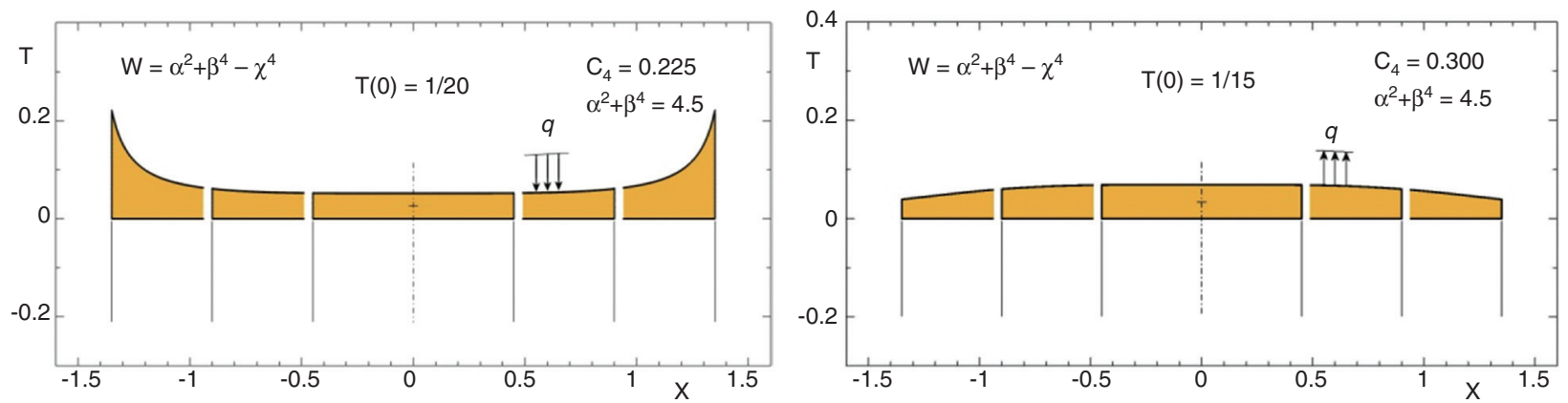

Figure 52 Thickness distributions $T(\chi)$ for a second-and fourth-degree flexure $W(\chi)$ along three cylindrical shells submitted to uniform load $q$. Opposite signs of $q$ - left and right figures - show the two families that generate the same flexure. The dimensionless constant $\beta$ originated from the requested flexure from optics. Dimensionless constant $\alpha$ is a free parameter to force getting a monotonic sign deformation and a practicable thickness distribution along $\chi$-axis. (The thickness variations are greatly exaggerated for clarity.) 
or compression of a tubular shell, caused by a uniform load $q$ applied at the surface of the shell, one can show that the radial thickness distribution $T(\chi)$ can be selected to remain of finite value. This avoids distributions, which exhibit local infinite thicknesses. For generating a given polynomial flexure along the shell, i.e., along its axis $\chi$, this theory provides the two solution families proposed by Lemaitre $[1,35]$ :

$\Rightarrow$ If the first family is represented by a load $q>0$ and thickness $T(\chi)$ from $\chi=0$ to outer ends $\pm \chi_{\max }$, then a second family exists with $q<0$ and $T(\chi)$ from $\chi=0$ to $\pm \chi_{\max }$.

From this law, one displays thickness distributions of two families, which generate a parabolic flexure $W\left(\chi^{2}\right)$ (curvature mode) and a fourth-degree flexure $W\left(\chi^{4}\right)$ by uniform loading $q$ positive and negative (Figure 52). A combined distribution of these thicknesses easily provides the convergence mode and stigmatism mode for the elasticity design of elongated ellipsoids usable as X-ray relay mirrors [1].

\subsection{X-ray tubular telescope mirrors and sine condition}

Future high angular resolution two-mirror tubular X-ray telescopes will probably evolve from the classical

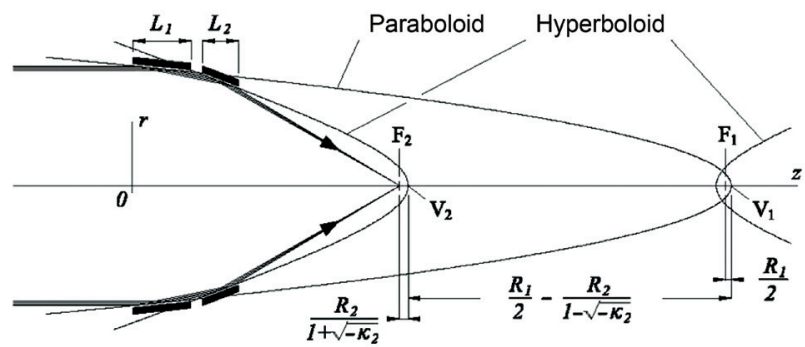

paraboloid-hyperboloid (PH) optical design (Figure 53) toward a Wolter-Schwarzchild (WS) design - as derived by Chase VanSpeybroeck [36] - which fully satisfies Abbe's sine condition (Figure 54). Owing to the extremely short wavelength, and the new telescope projects requiring a 0.1 arcsec resolution goal, it is obvious that the implementation of active optics will be necessary.

We have developed an elasticity theory of weakly conical shells $[1,35]$ and applied it to the optical design of such high-resolution telescope mirrors. Analytic results show that radial flexure distributions can be generated along the shell with a monotonic sign. This latter feature avoids the difficulty of a distribution with infinite thicknesses.

Let us represent the distribution of the radial flexural extension - or compression - $W(\chi)$ along the symmetry axis $\chi$ of the tube as the series

$$
W= \pm \alpha^{2}+\sum_{n=1,2,3 \ldots}^{N} A_{n} \chi^{n}
$$

where $\chi$ is the axis of the mirror, which ends at $[-\beta ; \beta], \alpha$, a constant to be set up for obtaining a monotonic sign of the flexure all along the mirror, and $A_{n}$, coefficients determined by the mirror shape deviation to a best fit cone or circle. The result of the weakly conical shell

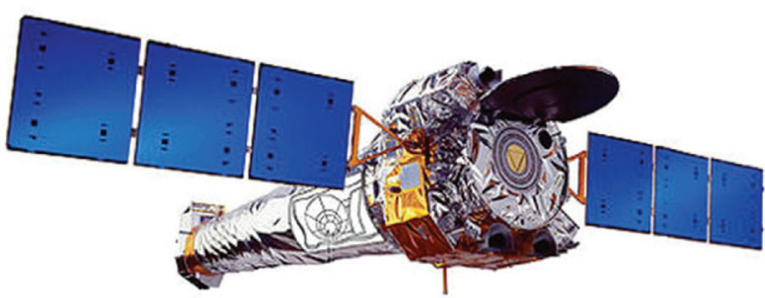

Figure 53 Left: Basic paraboloid-hyperboloid (PH) design of the two-mirror grazing incidence X-ray telescope. Right: Chandra X-ray Observatory launched in 1999 is a PH design (NASA).
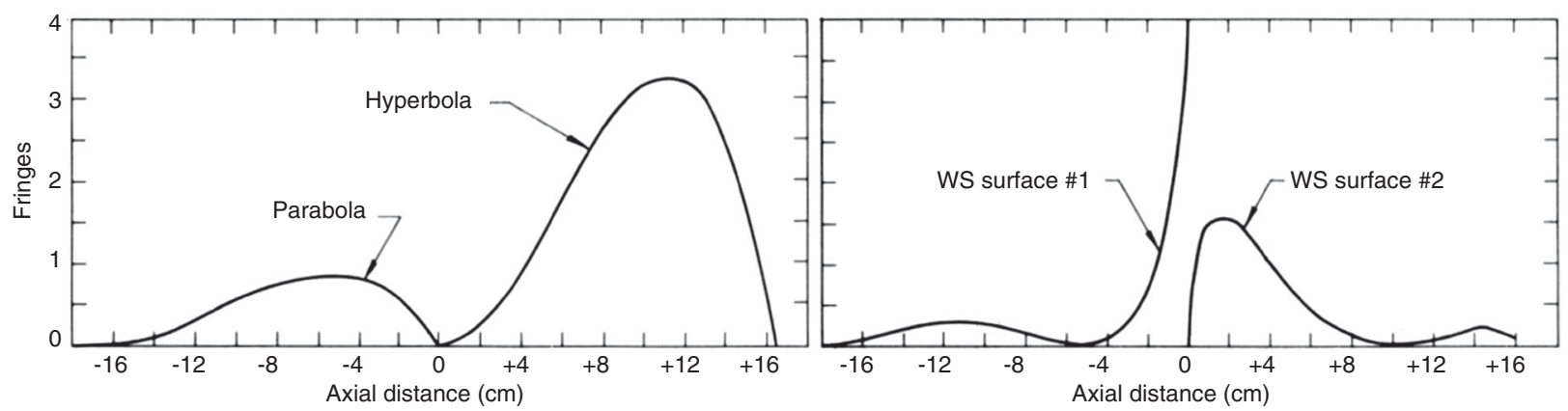

Figure 54 Comparison of two-mirror X-ray telescope designs: PH design vs. WS design. Mirror shapes with respect to best fit circle. WolterSchwarzschild (WS) form satisfies the sine condition (Chase and Van Speybroeck). 


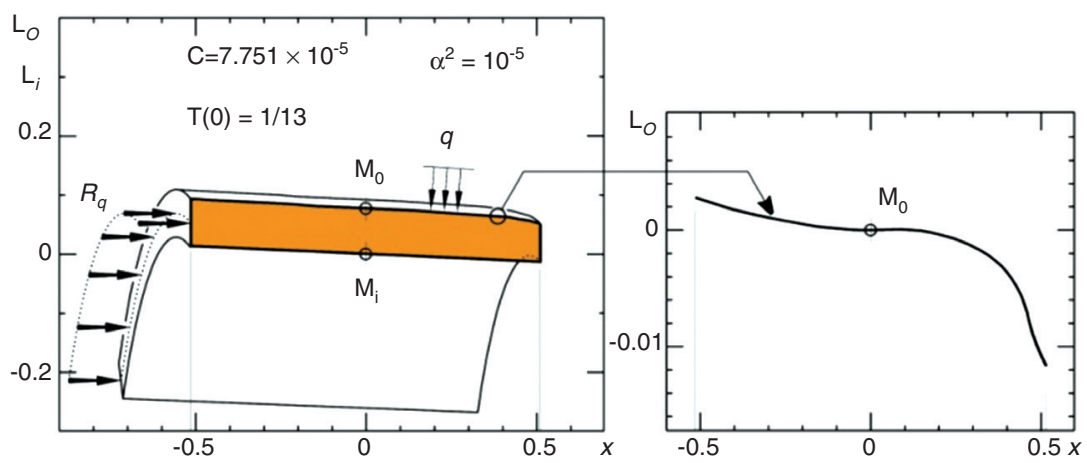

Figure 55 Weakly conical shell and thickness distribution $T(\chi)$ showing two inflexion points as for the optical shape of mirror \#1 of WS primary mirror (in Figure 54). Aspherization by uniform load $q$ from the best fit circle, here shown by the inner line $L_{i}$ of the section. The outer line $\mathrm{L}_{0}$ provides the thickness distribution $T(\chi)$. Note the axial reaction $R_{q}$ due to the uniform load $q$, which must be compensated.

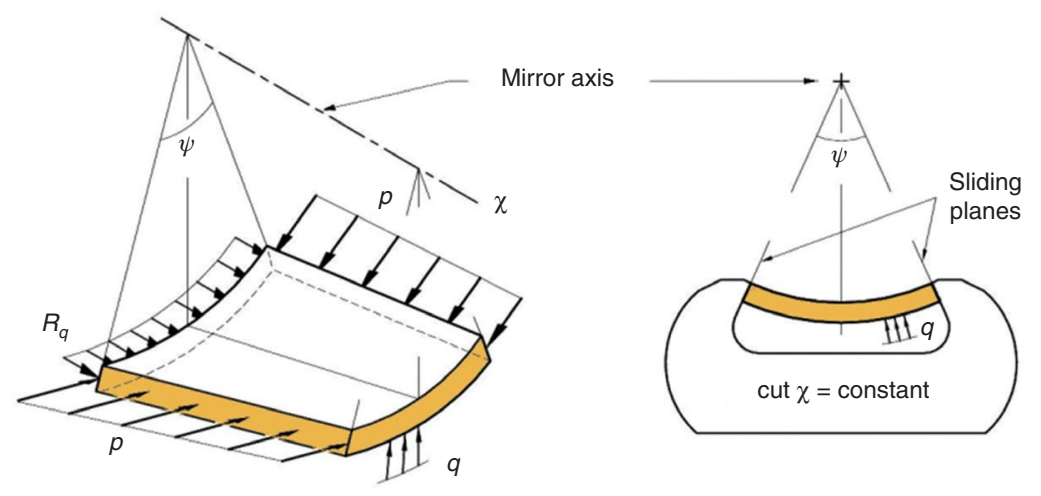

Figure 56 Left: X-ray telescope segment: configuration for aspherization by stress figuring and uniform loading. Right: Schematic harness satisfying the boundary conditions $\mathrm{C} 1, \mathrm{C} 2$, and $\mathrm{C} 3$.

theory provides the thickness $T(\chi)$ as easily derived from the flexure $W(\chi)$ by a linear product law (Lemaitre $[1,35]):$

$$
T(\chi) W(\chi)=C(1-2 i \chi) /\left(1-t_{0} / a_{0}\right), \quad \chi \in[-\beta, \beta]
$$

where $C$ is a constant (from load $q$, Young modulus $E$ ), $i$ is the mirror slope at $\chi=0, t_{0}$ is the shell thickness at $\chi=0, a_{0}$ is the shell radius, respectively, at $\chi=0$.

This law has been applied to the determination of the thickness distribution $T(\chi)$ for mirror \#1 of the WS design in Figure 54. The result is that the thickness profile $T(\chi)$ also shows two inflexion points very similar to that of the optical design (Figure 55).

In addition to obtaining smooth-surface X-ray mirrors by active optics methods, it has been emphasized by O'Dell et al. [37] and O'Dell [38] that in situ adaptive optics should be implemented to compensate for various deformation modes such as the residual deviation modes and some eigenmodes of a tubular mirror.

\subsection{Segmented X-ray telescope mirrors}

Similarly to large ground-based telescopes in the visible and infrared, future large space-based X-ray telescopes will require use of segmented and active mirrors. Current projects under investigation, e.g., Constellation X-ray Mission (NASA) and Xeus (ESA), mostly have architectures based on formation flyers.

From the above results of a monolithic tubular shell, active optics can be applied to the aspherization of an $\mathrm{X}$-ray segment of tubular telescope. The boundaries of this segment are derived from the stress distributions of a truncated part of a full shell [1] (Figure 56). Three boundary conditions must be satisfied for the aspherization of a segment through a harness.

This harness must fulfill the following properties:

C1: facets at $\pm \psi / 2$ supported by normal pressure $p$, C2: facets free to slide in the dihedral planes $\pm \psi / 2$, C3: receive reaction $R_{p}$ to load $q$ at largest facet end. 
The accurate achievement of these conditions does not present major technical difficulties.

\section{Conclusions}

The design of active optics systems may require the use of a large number of actuators such as for large existing monolithic telescope mirrors where the force actuators are uniformly distributed over the back surface of the mirror. However, active optics systems for smaller optics than $8 \mathrm{~m}$, which have to generate specific deformation modes, as presented here, could benefit from an optimal low number of actuators.

Since the pioneer work, almost 50 years ago, active optics methods have provided fascinating imaging quality in astronomy both in high angular resolution and in limiting magnitude detection. Active optics methods are key features for providing astronomers with powerful new telescopes and associated astronomical instrumentations.

\section{References}

[1] G. R. Lemaitre, in 'Astronomical Optics and Elasticity Theory Active Optics Methods', (Springer, Heidelberg, Germany, 3rd-print, 2012).

[2] E. E. Everhart, Appl. Opt. 5-5, 712 (1966).

[3] G. R. Lemaitre, Appl. Opt. 11-7, 1630 (1972).

[4] G. R. Lemaitre, C.R Acad. Sci. Paris 282-B, 87 (1976).

[5] G. R. Lemaitre, SPIE Proc. 4006, 192 (2000).

[6] M. Ferrari, Astron. Astrophys. 274, 12 (1994).

[7] M. Ferrari, Astron. Astrophys. 128, 221 (1998).

[8] F. Derie, SPIE Proc. 4006, 25 (2000).

[9] G. R. Lemaitre, Nouv. Rev. Opt. 5-6, 361 (1974).

[10] G. R. Lemaitre, C.R. Acad. Sci. Paris 288-B, 297 (1979).

[11] E. Hugot, M. Ferrari, A. Riccardi, M. Xompero, G.R. Lemaitre, et al., Astron. Astrophys. 538, A4 (2011).

[12] M. C. E. Huber, E. Jannitti, G. R. Lemaitre and G. Tondello, Appl. Opt. 20, 2139 (1981)

[13] E. Hugot. M. Ferrari, G.R. Lemaitre, F. Madec, S. Vives, et al., Appl. Opt. 48, 2932 (2009).

[14] N. J. Rumsey, in 'ICO 8 Proc. Optical Instruments and Techniques’, Ed. By Home Dickson (Oriel Press, 1969) pp. 514.
[15] A. Rakish, SPIE Proc. 5524, 101 (2004).

[16] G. R. Lemaitre, P. Montiel, P. Joulié, K. Dohlen and P. Lanzoni, Appl. Opt. 44, 7322 (2005).

[17] A. Labeyrie, Ann. Rev. Astron. Astrophys. 16, 77 (1978).

[18] G. R. Lemaitre, Meccanica, Springer 40-3, 233 (2005). Note: In this paper on nonaxisymmetric flexures, the author points out a correction, which should be noted in the book Theory of Plate and Shells by S. P. Timoshenko and S. Woinowsky-Krieger when representing the net shearing force $V_{r}$ with their sign convention. A similar sign incoherence on $V_{r}$ also occurs in the original paper by E. Riessner when elaborating the theory of shallow spherical shells and in several other classical publications on elasticity theory taking into account the effect of shear stress.

[19] J. Lubliner and J. E. Nelson, Appl. Opt. 19, 2332 (1980).

[20] J. E. Nelson, G. Gabor, L. K. Hunt, J. Lubliner and T. S. Mast, Appl. Opt. 19, 2341 (1980).

[21] R. N. Wilson, F. Franza and L. Noethe, J. Mod. Opt. 34, 485 (1987).

[22] R. N. Wilson, in 'Reflecting Telescope Optics', (Springer, Heidelberg, Germany, 1998).

[23] S.-g. Wang, D.-q. Su, Y.-q. Chu, X. Cui and Y.-n. Wang, Appl. Opt. 35, 5155 (1996).

[24] D.-q. Su, W.-j. Zou, Z.-c. Zhang, Y. Qu and S. Yang, SPIE Proc. 4003, 417 (2002).

[25] D.-q. Su and X. Cui, Chin. J. Astron. Astrophys. 4, 1 (2004).

[26] X. Cui, Y. Li, X. Ni, D. Yang, Z. Yao, et al., SPIE Proc. 4837, 628 (2002).

[27] X. Cui, D.-q. Su, G. Li, Z. Yao, Z. Zhang, et al., SPIE Proc. 5489, 974 (2004).

[28] M. Duban, Appl. Opt. 38, 1096 (1999).

[29] G. R. Lemaitre, ICLO XI, St. Petersburg, SPIE Proc. 5481, 70 (2004).

[30] M. Ferrari, E. Hugot, G. R. Lemaitre, C. du Jeu and J. -F. Carre, US Patent US-2009315202 (2009), European Patent EP-2144093 (2010).

[31] M. Laslandes, N. Rousselet, M. Ferrari, E. Hugot, J. Floriot, et al., SPIE Proc. 8169 (2011).

[32] B. Delabre, Astron. Astrophys., 487, 389 (2009).

[33] E. Vernet, M. Cayrel, N. Hubin, M. Mueller, R. Biasi et al., SPIE Proc. 8447 (2012).

[34] M. Cayrel, SPIE Proc. 8444-69 (2012).

[35] G. R. Lemaitre, SPIE Proc. 7011 (2008).

[36] R. C. Chase and L. P. VanSpeybroeck, Appl. Opt. 12, 1042 (1973).

[37] S. L. O'Dell, M. V. Gubarev et al., in 'Proc. of the International Workshop on Astronomical X-Ray Optics’, (Prague, Czech Republic, 2009).

[38] S. L. O'Dell, SPIE Proc. Adaptive X-ray Optics, 7803-15 (2009). 


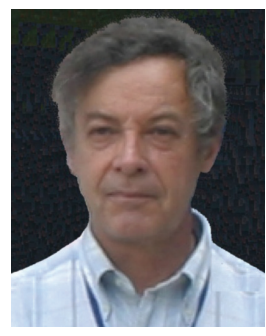

Gerard R. Lemaitre is an emeritus professor at Aix-Marseille University (AMU). His research interests are in the fields of active optics methods, elasticity theory, optical design for telescopes, and astronomical instrumentations. He develops active optics methods, discovering and making variable curvature mirrors, a two-zone loading method for making aspheric plates, optics replication method via active deformable masters for making toroid or planeapheric aberration-corrected gratings. He found the tulip form mirror class, cycloid-like form mirror class, vase form mirrors, and closed vase form mirrors for the aspherization by the elastic relaxation of telescope secondary mirrors and off-axis aberration-correcting mirrors. He is a holder of 10 patents and author of the book 'Astronomical Optics and Elasticity Theory,' Springer, 2010 (600p.). He has been leader for 30 years of the Marseille Observatory Optical Lab (LOOM) now at LAM. He established the optimal optical design to be given a reflective Schmidt system, was named an international member of the giant reflective Schmidt telescope LAMOST project committee by the Chinese Academy of Sciences - under operation at the Xinglong Station observatory of the NAOC - and of the 39-m ESE telescope committee for the E-ELT project by European Southern Observatory. 